\title{
Higher Haantjes Brackets and Integrability
}

\author{
Piergiulio Tempesta ${ }^{1,2}$ (]) , Giorgio Tondo ${ }^{3}$ \\ ${ }^{1}$ Departamento de Física Teórica, Facultad de Ciencias Físicas, \\ Universidad Complutense de Madrid, 28040 Madrid, Spain. \\ E-mail: p.tempesta@fis.ucm.es; piergiulio.tempesta@icmat.es \\ 2 Instituto de Ciencias Matemáticas, C/ Nicolás Cabrera, No 13-15, 28049 Madrid, Spain \\ 3 Dipartimento di Matematica e Geoscienze, Università degli Studi di Trieste, piaz.le Europa 1, 34127 Trieste, \\ Italy. E-mail: tondo@units.it
}

Received: 10 February 2021 / Accepted: 5 September 2021

Published online: 2 November 2021 - (C) The Author(s) 2021

\begin{abstract}
We propose a new, infinite class of brackets generalizing the FrölicherNijenhuis bracket. This class can be reduced to a family of generalized Nijenhuis torsions recently introduced. In particular, the Haantjes bracket, the first example of our construction, is relevant in the characterization of Haantjes moduli of operators. We also prove that the vanishing of a higher-level Nijenhuis torsion of an operator field is a sufficient condition for the integrability of its eigen-distributions. This result (which does not require any knowledge of the spectral properties of the operator) generalizes the celebrated Haantjes theorem. The same vanishing condition also guarantees that the operator can be written, in a local chart, in a block-diagonal form.
\end{abstract}

\section{Contents}

1. Introduction . . . . . . . . . . . . . . . . . . . . . . . . 1648

2. Preliminaries on the Nijenhuis and Haantjes Geometries . . . . . . . . . . 1650

3. Haantjes Brackets . . . . . . . . . . . . . . . . . 1651

3.1 The Frölicher-Nijenhuis bracket . . . . . . . . . . . . . . . . 1651

3.2 A new family of higher brackets . . . . . . . . . . . . . . . 1653

3.3 Haantjes brackets and Haantjes modules . . . . . . . . . . . . . . . 1656

3.3.1 The general case . . . . . . . . . . . . . . . . . . 1656

3.3.2 The semisimple, Abelian case . . . . . . . . . . . . . 1658

3.4 Spectral analysis . . . . . . . . . . . . . . . . . . . . . 1659

4. Generalized Nijenhuis Torsions and Haantjes Brackets for Nilcyclic Operators 1659

4.1 Triangular form of nilcyclic operators . . . . . . . . . . . . . . 1660

4.2 An open problem: a Jordan-Chevalley decomposition . . . . . . . . . 1662

4.3 Conjecture for higher Haantjes brackets . . . . . . . . . . . . . . . 1662

5. Frobenius Integrability and a Generalized Haantjes Theorem . . . . . . . . 1663

5.1 Integrability of eigen-distributions: necessary and sufficient conditions 1663

5.2 Main theorem . . . . . . . . . . . . . . . . . . 1664 
5.3 Block-diagonalization . . . . . . . . . . . . . . . . . 1666

5.4 A comparison with Haantjes's classical theorem . . . . . . . . . 1668

6. Appendix . . . . . . . . . . . . . . . . . . . . . . 1670

6.1 On the Haantjes bracket . . . . . . . . . . . . . . . . . . 1670

6.2 The Nijenhuis torsion evaluated over generalized eigenvectors . . . . 1670

6.3 Haantjes brackets evaluated over common eigenvectors . . . . . . 1670

Acknowledgements . . . . . . . . . . . . . . . . . 1671

References . . . . . . . . . . . . . . . . . . . . 1671

\section{Introduction}

In the last two decades, the study of the geometry of Nijenhuis and Haantjes tensors has experienced a resurgence of interest. The notion of Nijenhuis torsion was introduced in $[19,20]$ by A. Nijenhuis in his study of the integrability of eigen-distributions of operator fields with pointwise distinct eigenvalues.

In [10], the graded bracket nowadays called the Frölicher-Nijenhuis bracket was defined. This bracket is relevant in several geometric contexts, in particular the theory of almost-complex structures, as clarified by the Newlander-Nirenberg theorem $[12,18]$. Slightly before, in the seminal paper [11], J. Haantjes proposed the fundamental notion of torsion bearing his name. He proved that the vanishing of the Haantjes torsion of a $(1,1)$-tensor field is a necessary condition for the existence of an integrable frame of generalized eigenvectors. This condition is also sufficient in the case of pointwise semisimple operators.

Recently, new and conspicuous applications of Nijenhuis and Haantjes tensors have been found, for instance, in the characterization of integrable chains of partial differential equations of hydrodynamic type (see e.g. [4,9]) and in the study of infinite-dimensional integrable systems, in connection with the celebrated WDVV equations of associativity and the theory of Dubrovin-Frobenius manifolds [15-17]. In [22-25], the notion of Haantjes algebras and the related ones of $\omega \mathcal{H}$ and $P \mathcal{H}$ manifolds have been proposed as a natural setting for the formulation of the theory of finite-dimensional integrable Hamiltonian systems.

The aim of this work is twofold.

Our first goal is to introduce a new, infinite class of brackets that generalize the Frölicher-Nijenhuis one. The first representative of our class coincides with it; the second one is already a new example, the Haantjes bracket $\mathcal{H}_{\boldsymbol{A}, \boldsymbol{B}}(X, Y)$. By means of a recursive procedure, we also define a "tower" of infinitely many novel higher-level brackets. Here, "bracket" is used to emphasize their dependence on a pair $(\boldsymbol{A}, \boldsymbol{B})$ of $(1,1)$-tensor fields.

A simple reduction of this family, obtained when each representative depends on a pair $(\boldsymbol{A}, \boldsymbol{A})$ of copies of the same operator field, coincides (up to a constant) with the family of generalized torsions defined independently (and from a different perspective) by Kosmann-Schwarzbach in [13] and by ourselves in an early, preprint 2017 version of [23].

Within this framework, we wish to study the geometry of very general families of operators, as the triangularizable ones, that (except in very specific cases) have nonvanishing Haantjes torsion.

We have ascertained the geometric relevance of our higher brackets in several important situations. Precisely, as stated in Theorem 23, given two commutative semisimple operators, they generate a Haantjes module if and only if their Haantjes bracket vanishes. Also, further algebraic properties of this bracket have been studied. 
Our second goal is to clarify the geometric meaning of the "generalized Nijenhuis torsions" of higher level introduced, with different formulations, in [13,23]. In Sect. 5 (Proposition 26 and Corollary 27) we prove that the vanishing of the generalized Nijenhuis torsion $\tau_{A}^{(n-1)}(X, Y)=0$ of level $(n-1)$ of a nilcyclic (i.e. both nilpotent and cyclic) operator field $\boldsymbol{A}$ on a manifold of dimension $n$ is necessary for the existence of a local chart where $\boldsymbol{A}$ takes a triangular form (see Eq. (46)).

The main theorem of the present work, Theorem 40 of Section 6, concerns the integrability properties of the generalized eigen-distributions (i.e., distributions of generalized eigenvectors) of an operator field. A seminal result, due to Haantjes [11], states that in the case of a semisimple operator field, a necessary and sufficient condition for the Frobenius integrability of its eigen-distributions of constant rank is that its Haantjes tensor identically vanishes. However, in the general case of a non-semisimple operator, the previous condition is only sufficient. Thus, for the infinite class of operators whose Haantjes tensor is not vanishing, no conclusion can be drawn about integrability of their eigen-distributions.

Our main theorem fills this gap. Indeed, we shall prove that the vanishing of a generalized Nijenhuis torsion $\tau_{A}^{(m)}(X, Y)$ of level $m$ for some integer $m \geq 1$ provides us with a sufficient condition for the integrability of the generalized eigen-distributions of a given operator field $\boldsymbol{A}$. In addition, it ensures the integrability of all of their direct sums. Thus, we are able to construct a tensorial test for the Frobenius integrability of a very large class of operator fields, which significantly extends the applicability of the original Haantjes's torsion criterion.

The interest of our result, in the spirit of the Haantjes theorem, relies crucially on the fact that, in order to ascertain the integrability properties of a given operator, no knowledge a priori of the spectrum of this operator nor of its eigen-distributions is required.

At the same time, for completeness we also provide necessary and sufficient conditions for the Frobenius integrability of the eigen-distributions of an operator field in terms of generalized tensors of higher level, assuming the knowledge of its eigen-distributions (see Corollary 34). Under this hypothesis, we show that the Nijenhuis torsion as well as any of the higher level ones allow us to characterize integrable eigen-distributions of operator fields.

An important consequence of Theorem 40 (see Proposition 43) is the fact that an operator with a vanishing generalized Nijenhuis torsion (for some $m \geq 1$ ) admits a local coordinate chart where it takes a block-diagonal form.

In short, the body of results proposed indicates that all of the infinitely many higherlevel tensors introduced possess a geometric meaning and are relevant in applicative contexts.

An open problem we propose in Sect. 5 is the decomposition of a generic operator field as the sum of a diagonal operator and an operator whose generalized Nijenhuis torsion (of a suitable level) vanishes.

We also believe that the theory of higher brackets defined in this work could play a significant role, more generally, in the theory of integrable systems, for instance in the study of generic hydrodynamic-type systems, not possessing Riemann invariants. For instance, a potentially interesting area is the study of equations of hydrodynamic type in $(2+1)$ dimensions, namely, equations of the form $u_{t}=\boldsymbol{A}(u) u_{x}+\boldsymbol{B}(u) u_{y}$, where $\boldsymbol{A}(u)$ and $\boldsymbol{B}(u)$ are operator fields which not necessarily commute [8]. It would be interesting, for instance, to classify the pairs of operators $(\boldsymbol{A}(u), \boldsymbol{B}(u))$ appearing in the 
theory of hydrodynamic-type systems by means of suitable tensor conditions ensuring integrability (some results are presented in Sect. 3.3.1).

\section{Preliminaries on the Nijenhuis and Haantjes Geometries}

In this section, we shall review some basic notions concerning the geometry of Nijenhuis and Haantjes torsions, following the original papers $[10,11,19]$. Here we shall focus only on the aspects of the theory which are relevant for the subsequent discussion.

Let $M$ be a differentiable manifold, $\mathfrak{X}(M)$ the Lie algebra of all smooth vector fields on $M$ and $\boldsymbol{A}: \mathfrak{X}(M) \rightarrow \mathfrak{X}(M)$ be a smooth $(1,1)$-tensor field (namely, an operator field). For the sake of simplicity, from now on the expressions "tensor fields" and "operator fields" will be abbreviated to tensors and operators. In the following, all tensors will be considered to be smooth.

Definition 1. The Nijenhuis torsion of $\boldsymbol{A}$ is the vector-valued 2-form defined by

$$
\tau_{\boldsymbol{A}}(X, Y):=\boldsymbol{A}^{2}[X, Y]+[\boldsymbol{A} X, \boldsymbol{A} Y]-\boldsymbol{A}([X, \boldsymbol{A} Y]+[\boldsymbol{A X}, Y]),
$$

where $X, Y \in \mathfrak{X}(M)$ and [ , ] denotes the Lie bracket of two vector fields.

Definition 2. The Haantjes torsion of $\boldsymbol{A}$ is the vector-valued 2-form defined by

$$
\mathcal{H}_{A}(X, Y):=A^{2} \tau_{A}(X, Y)+\tau_{A}(A X, A Y)-A\left(\tau_{A}(X, A Y)+\tau_{A}(A X, Y)\right)
$$

Definition 3. A Haantjes (Nijenhuis) operator is a (1,1)-tensor whose Haantjes (Nijenhuis) torsion identically vanishes.

A simple, relevant case of Haantjes operator is that of a tensor $\boldsymbol{A}$ which takes a diagonal form in a local chart $\boldsymbol{x}=\left(x^{1}, \ldots, x^{n}\right)$ :

$$
\boldsymbol{A}(\boldsymbol{x})=\sum_{i=1}^{n} \lambda_{i}(\boldsymbol{x}) \frac{\partial}{\partial x^{i}} \otimes \mathrm{d} x^{i},
$$

where $\lambda_{i}(\boldsymbol{x}):=\lambda_{i}^{i}(\boldsymbol{x})$ are the eigenvalues of $\boldsymbol{A}$ and $\left(\frac{\partial}{\partial x^{1}}, \ldots, \frac{\partial}{\partial x^{n}}\right)$ are the fields forming the so called natural frame associated with the local chart $\left(x^{1}, \ldots, x^{n}\right)$. As is well known, the Haantjes torsion of the diagonal operator (3) vanishes.

We also recall that two frames $\left\{X_{1}, \ldots, X_{n}\right\}$ and $\left\{Y_{1}, \ldots, Y_{n}\right\}$ are said to be equivalent if $n$ nowhere vanishing smooth functions $f_{i}$ exist, such that

$$
X_{i}=f_{i}(\boldsymbol{x}) Y_{i}, \quad i=1, \ldots, n .
$$

Definition 4. [1] An integrable frame is a reference frame equivalent to a natural frame.

Remark 5. We wish to point out that the adjectives "diagonalizable" and "semisimple" are both used in the literature, sometimes interchangeably. From now on, we shall call diagonalizable an operator which takes a diagonal form in a natural reference frame (as in formula (3)), whereas we shall say that an operator is pointwise semisimple (or semisimple tout court) if it admits a local reference frame (not necessarily natural, nor integrable) in which it takes a diagonal form. Diagonalizable operators are obviously semisimple; the converse statement is not true in general. Historically, the problem addressed by Nijenhuis and Haantjes was to ascertain whether a local reference frame constructed out of the eigenvectors of an operator is integrable or not. 
It is interesting to observe that the algebraic properties of Haantjes operators are different, and sometimes richer that those of Nijenhuis operators. A useful result is the following (hereafter, $\boldsymbol{I}: \mathfrak{X}(M) \rightarrow \mathfrak{X}(M)$ will denote the identity operator).

Proposition 6. [2] Let $\boldsymbol{A}$ be a (1,1)-tensor. The following identity holds:

$$
\mathcal{H}_{f+g A}(X, Y)=g^{4} \mathcal{H}_{A}(X, Y)
$$

where $f, g: M \rightarrow \mathbb{R}$ are $C^{\infty}(M)$ functions.

Proof. See Proposition 1, p. 255 of [2].

Interestingly enough, such a simple property does not hold in the case of a Nijenhuis operator.

Many more examples of Haantjes operators, relevant in classical mechanics and in Riemannian geometry, can be found for instance in [21-25].

\section{Haantjes Brackets}

Let $M$ be a differentiable manifold and $\boldsymbol{A}, \boldsymbol{B}: \mathfrak{X}(M) \rightarrow \mathfrak{X}(M)$ be two operators.

\subsection{The Frölicher-Nijenhuis bracket.}

Definition 7. [10] The Frölicher-Nijenhuis bracket of $\boldsymbol{A}$ and $\boldsymbol{B}$ is the vector-valued 2-form given by ${ }^{1}$

$$
\begin{aligned}
& \llbracket \boldsymbol{A}, \boldsymbol{B} \rrbracket(X, Y):=(\boldsymbol{A} \boldsymbol{B}+\boldsymbol{B} \boldsymbol{A})[X, Y]+[\boldsymbol{A} X, \boldsymbol{B} Y]+[\boldsymbol{B} X, \boldsymbol{A} Y] \\
& -\boldsymbol{A}([X, \boldsymbol{B} Y]+[\boldsymbol{B} X, Y])-\boldsymbol{B}([X, \boldsymbol{A} Y]+[\boldsymbol{A} X, Y]), \quad X, Y \in \mathfrak{X}(M) .
\end{aligned}
$$

The local expression of the components of the Frölicher-Nijenhuis bracket reads

$$
\llbracket \boldsymbol{A}, \boldsymbol{B} \rrbracket_{j k}^{i}=\sum_{l=1}^{n}\left(\boldsymbol{A}_{[j}^{l} \partial_{|l|} \boldsymbol{B}_{k]}^{i}-\boldsymbol{A}_{l}^{i} \partial_{[j} \boldsymbol{B}_{k]}^{l}+\boldsymbol{B}_{[j}^{l} \partial_{|l|} \boldsymbol{A}_{k]}^{i}-\boldsymbol{B}_{l}^{i} \partial_{[j} \boldsymbol{A}_{k]}^{l}\right) .
$$

This bracket has relevant geometric applications [18], in particular in the theory of almost-complex structures and in the detection of obstructions to integrability [12]. The bracket is symmetric and $\mathbb{R}$-linear (but not $C^{\infty}(M)$-linear) in $\boldsymbol{A}$ and $\boldsymbol{B}$. In fact, it satisfies the identity

$$
\begin{aligned}
\llbracket f \boldsymbol{A}, g \boldsymbol{B} \rrbracket(X, Y)= & f g \llbracket \boldsymbol{A}, \boldsymbol{B} \rrbracket(X, Y)-g\left(\mathbf{T}(\boldsymbol{B}, \boldsymbol{A})-\mathbf{T}^{T}(\boldsymbol{B}, \boldsymbol{A})(\mathrm{d} f, X, Y)\right) \\
& -f\left(\mathbf{T}(\boldsymbol{A}, \boldsymbol{B})-\mathbf{T}^{T}(\boldsymbol{A}, \boldsymbol{B})(\mathrm{d} g, X, Y)\right) .
\end{aligned}
$$

Here T $(\boldsymbol{A}, \boldsymbol{B}): \mathfrak{X}^{*}(M) \times \mathfrak{X}(M) \times \mathfrak{X}(M) \rightarrow \mathfrak{X}(M)$ is the vector-valued 3-tensor defined by

$$
\mathbf{T}(\boldsymbol{A}, \boldsymbol{B})(\alpha, X, Y):=(\boldsymbol{I} \otimes \boldsymbol{A} \boldsymbol{B}-\boldsymbol{A} \otimes \boldsymbol{B})(\alpha, X, Y) .
$$

\footnotetext{
${ }^{1}$ For sake of clarity, in this article we have renounced to the usual unified notation $[\cdot, \cdot]$ which, depending on the context, should stand for both the standard Lie bracket of vector fields and the Frölicher-Nijenhuis bracket of operators. Instead, we have preferred to maintain the symbol $\llbracket \cdot, \cdot \rrbracket$ for the Frölicher-Nijenhuis bracket and to introduce the notation $[\cdot, \cdot]$ for the Lie bracket of two vector fields as well as the commutator of two operators.
} 
We shall denote by $\mathbf{T}^{T}(\alpha, X, Y):=\mathbf{T}(\alpha, Y, X)$ the transposed of $\mathbf{T}$ w.r.t. the last two arguments. We also recall that for each operator $\boldsymbol{A}, \boldsymbol{B}$, and for all $\alpha \in \mathfrak{X}^{*}(M), X, Y \in$ $\mathfrak{X}(M)$,

$$
(\boldsymbol{A} \otimes \boldsymbol{B})(\alpha, X, Y)=\langle\alpha, \boldsymbol{A} X\rangle \boldsymbol{B} Y .
$$

Note that for each operator $\boldsymbol{B}: \mathfrak{X}(M) \rightarrow \mathfrak{X}(M)$, we have

$$
\llbracket \boldsymbol{I}, \boldsymbol{B} \rrbracket(X, Y)=\mathbf{0}, \quad \forall X, Y \in \mathfrak{X}(M) .
$$

Choosing $\boldsymbol{A}=\boldsymbol{B}$ in Eq. (5), one gets twice the Nijenhuis torsion:

$$
\llbracket \boldsymbol{A}, \boldsymbol{A} \rrbracket(X, Y)=2 \tau_{\boldsymbol{A}}(X, Y) .
$$

For all $f, g \in C^{\infty}(M)$, the following identity holds:

$$
\begin{aligned}
\tau_{f \boldsymbol{A}+g \boldsymbol{B}}(X, Y)= & f^{2} \tau_{\boldsymbol{A}}(X, Y)-f\left(\mathbf{T}(\boldsymbol{A}, \boldsymbol{A})-\mathbf{T}^{T}(\boldsymbol{A}, \boldsymbol{A})\right)(\mathrm{d} f, X, Y)+g^{2} \tau_{\boldsymbol{B}}(X, Y) \\
& -g\left(\mathbf{T}(\boldsymbol{B}, \boldsymbol{B})-\mathbf{T}^{T}(\boldsymbol{B}, \boldsymbol{B})\right)(\mathrm{d} g, X, Y)+f g \llbracket \boldsymbol{A}, \boldsymbol{B} \rrbracket(X, Y) \\
& -f\left(\mathbf{T}(\boldsymbol{A}, \boldsymbol{B})-\mathbf{T}^{T}(\boldsymbol{A}, \boldsymbol{B})\right)(\mathrm{d} g, X, Y) \\
& -g\left(\mathbf{T}(\boldsymbol{B}, \boldsymbol{A})-\mathbf{T}^{T}(\boldsymbol{B}, \boldsymbol{A})\right)(\mathrm{d} f, X, Y) .
\end{aligned}
$$

This identity allows us to characterize modules of Nijenhuis operators.

Proposition 8. Let $\boldsymbol{A}$ and $\boldsymbol{B}$ be two Nijenhuis operators. They generate a module of Nijenhuis operators if and only if the following conditions are fulfilled

$$
\begin{gathered}
\llbracket \boldsymbol{A}, \boldsymbol{B} \rrbracket=\mathbf{0}, \\
\mathbf{T}(\boldsymbol{A}, \boldsymbol{A})=\mathbf{T}^{T}(\boldsymbol{A}, \boldsymbol{A}), \quad \mathbf{T}(\boldsymbol{B}, \boldsymbol{B})=\mathbf{T}^{T}(\boldsymbol{B}, \boldsymbol{B}), \\
\mathbf{T}(\boldsymbol{A}, \boldsymbol{B})=\mathbf{T}^{T}(\boldsymbol{A}, \boldsymbol{B}), \quad \mathbf{T}(\boldsymbol{B}, \boldsymbol{A})=\mathbf{T}^{T}(\boldsymbol{B}, \boldsymbol{A}) .
\end{gathered}
$$

In particular, they generate a vector space of Nijenhuis operators if and only if their Frölicher-Nijenhuis bracket identically vanishes.

Example. (i) The couple of Nijenhuis operators $\boldsymbol{A}=f(\boldsymbol{x}) \frac{\partial}{\partial x^{1}} \otimes \mathrm{d} x^{n}, \boldsymbol{B}=g(\boldsymbol{x}) \frac{\partial}{\partial x^{1}} \otimes$ $\mathrm{d} x^{n}$ satisfy conditions (11)-(13); then, they generate a module of Nijenhuis operators.

(ii) The couple of Nijenhuis operators $\boldsymbol{A}=f\left(x^{i}\right) \frac{\partial}{\partial x^{i}} \otimes \mathrm{d} x^{i}, \boldsymbol{B}=g\left(x^{k}\right) \frac{\partial}{\partial x^{k}} \otimes \mathrm{d} x^{k}$, whose Frölicher-Nijenhuis bracket vanishes, generate a vector space of Nijenhuis operators. 
3.2. A new family of higher brackets. Hereafter, we shall present the main algebraic construction of this work, namely the recursive definition of an infinite "tower" of new brackets of couples of operators.

Definition 9. Let $M$ be a differentiable manifold of dimension $n$ and let $\boldsymbol{A}, \boldsymbol{B}: \mathfrak{X}(M) \rightarrow$ $\mathfrak{X}(M)$ be two $(1,1)$-tensors. The Haantjes bracket of level $m \in \mathbb{N} \backslash\{0\}$ of $\boldsymbol{A}$ and $\boldsymbol{B}$ is the vector-valued 2-form defined, for any $X, Y \in \mathfrak{X}(M)$, by the relations

$$
\mathcal{H}_{\boldsymbol{A}, \boldsymbol{B}}^{(1)}(X, Y):=\llbracket \boldsymbol{A}, \boldsymbol{B} \rrbracket(X, Y)
$$

and

$$
\begin{aligned}
\mathcal{H}_{\boldsymbol{A}, \boldsymbol{B}}^{(m)}(X, Y):= & (\boldsymbol{A} \boldsymbol{B}+\boldsymbol{B} \boldsymbol{A}) \mathcal{H}_{\boldsymbol{A}, \boldsymbol{B}}^{(m-1)}(X, Y)+\mathcal{H}_{\boldsymbol{A}, \boldsymbol{B}}^{(m-1)}(\boldsymbol{A} X, \boldsymbol{B} Y)+\mathcal{H}_{\boldsymbol{A}, \boldsymbol{B}}^{(m-1)}(\boldsymbol{B} X, \boldsymbol{A} Y) \\
& -\boldsymbol{A}\left(\mathcal{H}_{\boldsymbol{A}, \boldsymbol{B}}^{(m-1)}(X, \boldsymbol{B} Y)+\mathcal{H}_{\boldsymbol{A}, \boldsymbol{B}}^{(m-1)}(\boldsymbol{B} X, Y)\right) \\
& -\boldsymbol{B}\left(\mathcal{H}_{\boldsymbol{A}, \boldsymbol{B}}^{(m-1)}(X, \boldsymbol{A} Y)+\mathcal{H}_{\boldsymbol{A}, \boldsymbol{B}}^{(m-1)}(\boldsymbol{A} X, Y)\right), \quad m \geq 2 .
\end{aligned}
$$

None of these brackets for $m \geq 2$ is $\mathbb{R}$-linear in $\boldsymbol{A}$ and $\boldsymbol{B}$; however, they are symmetric in the interchange of $\boldsymbol{A}$ and $\boldsymbol{B}$.

The following statement can be useful for computational purposes.

Lemma 10. The expression in local coordinates of the Haantjes brackets of level $m$, for $m \geq 2$, reads

$$
\begin{aligned}
\left(\mathcal{H}_{\boldsymbol{A}, \boldsymbol{B}}^{(m)}\right)_{j k}^{i}= & \sum_{\alpha, \beta=1}^{n}\left(\boldsymbol{A}_{\alpha}^{i} \boldsymbol{B}_{\beta}^{\alpha}\left(\mathcal{H}_{\boldsymbol{A}, \boldsymbol{B}}^{(m-1)}\right)_{j k}^{\beta}+\boldsymbol{B}_{\alpha}^{i} \boldsymbol{A}_{\beta}^{\alpha}\left(\mathcal{H}_{\boldsymbol{A}, \boldsymbol{B}}^{(m-1)}\right)_{j k}^{\beta}+\left(\mathcal{H}_{\boldsymbol{A}, \boldsymbol{B}}^{(m-1)}\right)_{\alpha \beta}^{i} \boldsymbol{A}_{j}^{\alpha} \boldsymbol{B}_{k}^{\beta}\right. \\
& +\left(\mathcal{H}_{\boldsymbol{A}, \boldsymbol{B}}^{(m-1)}\right)_{\alpha \beta}^{i} \boldsymbol{B}_{j}^{\alpha} \boldsymbol{A}_{k}^{\beta}-\boldsymbol{A}_{\alpha}^{i}\left(\left(\mathcal{H}_{\boldsymbol{A}, \boldsymbol{B}}^{(m-1)}\right)_{j \beta}^{\alpha} \boldsymbol{B}_{k}^{\beta}+\left(\mathcal{H}_{\boldsymbol{A}, \boldsymbol{B}}^{(m-1)}\right)_{\beta k}^{\alpha} \boldsymbol{B}_{j}^{\beta}\right) \\
& \left.-\boldsymbol{B}_{\alpha}^{i}\left(\left(\mathcal{H}_{\boldsymbol{A}, \boldsymbol{B}}^{m-1}\right)_{j \beta}^{\alpha} \boldsymbol{A}_{k}^{\beta}+\left(\mathcal{H}_{\boldsymbol{A}, \boldsymbol{B}}^{(m-1)}\right)_{\beta k}^{\alpha} \boldsymbol{A}_{j}^{\beta}\right)\right) .
\end{aligned}
$$

Proof. This formula comes directly from the expression in local coordinates of the Frölicher-Nijenhuis bracket (6), applied to the recursive formula (14).

If we take $\boldsymbol{A}=\boldsymbol{B}$, then the previous family of brackets reduces to the generalized torsions proposed independently in [13] and in [23]. Here we remind the main definition of that construction, according to the formulation proposed in [23], since it will be crucial in the subsequent discussion.

Definition 11. Let $\boldsymbol{A}: \mathfrak{X}(M) \rightarrow \mathfrak{X}(M)$ be a $(1,1)$-tensor. The generalized Nijenhuis torsion of $\boldsymbol{A}$ of level $m$, for each integer $m \geq 1$, is the vector-valued 2 -form defined by

$$
\begin{aligned}
\tau_{\boldsymbol{A}}^{(m)}(X, Y):= & \frac{1}{2^{m}} \mathcal{H}_{\boldsymbol{A}, \boldsymbol{A}}^{(m)}(X, Y)=\boldsymbol{A}^{2} \tau_{\boldsymbol{A}}^{(m-1)}(X, Y)+\tau_{\boldsymbol{A}}^{(m-1)}(\boldsymbol{A} X, \boldsymbol{A} Y) \\
& -\boldsymbol{A}\left(\tau_{\boldsymbol{A}}^{(m-1)}(X, \boldsymbol{A} Y)+\tau_{\boldsymbol{A}}^{(m-1)}(\boldsymbol{A} X, Y)\right), \quad X, Y \in \mathfrak{X}(M) .
\end{aligned}
$$

Here the notation $\tau_{\boldsymbol{A}}^{(0)}(X, Y):=[X, Y], \tau_{\boldsymbol{A}}^{(1)}(X, Y):=\tau_{\boldsymbol{A}}(X, Y)$ and $\tau_{\boldsymbol{A}}^{(2)}(X, Y):=$ $\mathcal{H}_{A}(X, Y)$ is used. 
We also remind a useful formula, proved in [13] (Section 4.6), by means of a suitable polynomial representation of $(1,2)$-tensors:

$$
\tau_{\boldsymbol{A}}^{(m)}(X, Y)=\sum_{p=0}^{m} \sum_{q=0}^{m}(-1)^{2 m-p-q}\left(\begin{array}{c}
m \\
p
\end{array}\right)\left(\begin{array}{c}
m \\
q
\end{array}\right) \boldsymbol{A}^{p+q}\left[\boldsymbol{A}^{m-p} X, \boldsymbol{A}^{m-q} Y\right] .
$$

Alternatively, this formula can also be proved by induction over $m$.

Hereafter, we shall discuss some relevant properties of the new brackets (14).

Lemma 12. Let $M$ be a differentiable manifold and $\boldsymbol{A}, \boldsymbol{B}: \mathfrak{X}(M) \rightarrow \mathfrak{X}(M)$ be two operators. For any $X, Y \in \mathfrak{X}(M)$, we have

$$
\mathcal{H}_{\boldsymbol{I}, \boldsymbol{B}}^{(m)}(X, Y)=\mathbf{0}, \quad m \in \mathbb{N} \backslash\{0\} .
$$

Moreover, if $[\boldsymbol{A}, \boldsymbol{B}]:=\boldsymbol{A} \boldsymbol{B}-\boldsymbol{B} \boldsymbol{A}=\mathbf{0}$, we have

$$
\mathcal{H}_{f \boldsymbol{A}, g \boldsymbol{B}}^{(m)}(X, Y)=f^{m} g^{m} \mathcal{H}_{\boldsymbol{A}, \boldsymbol{B}}^{(m)}(X, Y), \quad m \in \mathbb{N} \backslash\{0,1\} .
$$

Consequently,

$$
\mathcal{H}_{f \boldsymbol{I}, \boldsymbol{B}}^{(m)}(X, Y)=\mathbf{0} .
$$

Proof. Relation (18) is obtained by induction over $m$, starting with the case $m=1$ already stated in Eq. (9). Similarly, property (19) can be proved by induction over $m \geq 2$; the case $m=2$ simply requires a direct calculation. Equation (20) is an immediate consequence of Eqs. (18) and (19).

Proposition 13. Let $M$ be a differentiable manifold and $\boldsymbol{A}, \boldsymbol{B}: \mathfrak{X}(M) \rightarrow \mathfrak{X}(M)$ two commuting operators. For any $f, g, h, k \in C^{\infty}(M), X, Y \in \mathfrak{X}(M)$ and for each integer $m \geq 2$, we have

$$
\mathcal{H}_{f \boldsymbol{I}+g \boldsymbol{A}, h \boldsymbol{I}+k \boldsymbol{B}}^{(m)}(X, Y)=g^{m} k^{m} \mathcal{H}_{\boldsymbol{A}, \boldsymbol{B}}^{(m)}(X, Y) .
$$

Proof. The formula can be proved by induction over $m$, starting with the case $h=0$ and $k=1$. Then, the result follows as a consequence of the symmetry w.r.t. the interchange of the first and second operator.

Corollary 14. Let $\boldsymbol{A}: \mathfrak{X}(M) \rightarrow \mathfrak{X}(M)$ be an operator. Then, for all $f \in C^{\infty}(M)$ the relations

$$
\begin{aligned}
\tau_{\boldsymbol{I}}^{(m)}(X, Y) & =\mathbf{0}, \quad m \in \mathbb{N} \backslash\{0\} \\
\tau_{f \boldsymbol{I}+g \boldsymbol{A}}^{(m)}(X, Y) & =g^{2 m} \tau_{\boldsymbol{A}}^{(m)}(X, Y), \quad m \in \mathbb{N} \backslash\{0,1\} .
\end{aligned}
$$

hold.

Proof. Equations (22) and (23) are obtained by choosing $g=1, h=f, k=1, \boldsymbol{B}=\boldsymbol{A}$ into Eq. (21).

The value $m=1$ has been excluded in Eq. (23), since for this case a separate formula for the Nijenhuis torsion holds:

$$
\tau_{g \boldsymbol{A}}(X, Y)=g^{2} \tau_{\boldsymbol{A}}(X, Y)-g\left(\mathbf{T}(\boldsymbol{A}, \boldsymbol{A})-\mathbf{T}^{T}(\boldsymbol{A}, \boldsymbol{A})\right)(\mathrm{d} g, X, Y) .
$$

This equation can be easily obtained from Eq. (7), choosing $f=g$ and $\boldsymbol{B}=\boldsymbol{A}$.

Let us consider in more detail the properties of the Haantjes bracket of level $m=2$ of two arbitrary commuting operators. Hereafter the notation $\mathcal{H}_{\boldsymbol{A}, \boldsymbol{B}}(X, Y):=\mathcal{H}_{\boldsymbol{A}, \boldsymbol{B}}^{(2)}(X, Y)$ will be used. 
Proposition 15. Let $M$ be a differentiable manifold, $f, g \in C^{\infty}(M)$ and let $\boldsymbol{A}, \boldsymbol{B}$ : $\mathfrak{X}(M) \rightarrow \mathfrak{X}(M)$ be two $(1,1)$-tensors. Then, the following identity holds:

$$
\begin{aligned}
& \mathcal{H}_{f \boldsymbol{A}, g \boldsymbol{B}}(X, Y)=f^{2} g^{2} \mathcal{H}_{\boldsymbol{A}, \boldsymbol{B}}(X, Y) \\
&+ f g^{2}(\boldsymbol{I} \otimes[\boldsymbol{A}, \boldsymbol{B}[\boldsymbol{A}, \boldsymbol{B}]]-\boldsymbol{B} \otimes[\boldsymbol{A},[\boldsymbol{A}, \boldsymbol{B}]]-\boldsymbol{A} \otimes \boldsymbol{B}[\boldsymbol{A}, \boldsymbol{B}]+\boldsymbol{B} \boldsymbol{A} \otimes[\boldsymbol{A}, \boldsymbol{B}]) \\
&(\mathrm{d} f, X, Y) \\
&- f g^{2}(\boldsymbol{I} \otimes[\boldsymbol{A}, \boldsymbol{B}[\boldsymbol{A}, \boldsymbol{B}]]-\boldsymbol{B} \otimes[\boldsymbol{A},[\boldsymbol{A}, \boldsymbol{B}]]-\boldsymbol{A} \otimes \boldsymbol{B}[\boldsymbol{A}, \boldsymbol{B}]+\boldsymbol{B} \boldsymbol{A} \otimes[\boldsymbol{A}, \boldsymbol{B}]) \\
&(\mathrm{d} f, Y, X) \\
&+ f^{2} g(\boldsymbol{I} \otimes[\boldsymbol{B}, \boldsymbol{A}[\boldsymbol{B}, \boldsymbol{A}]]-\boldsymbol{A} \otimes[\boldsymbol{B},[\boldsymbol{B}, \boldsymbol{A}]]-\boldsymbol{B} \otimes \boldsymbol{A}[\boldsymbol{B}, \boldsymbol{A}]+\boldsymbol{A B} \otimes[\boldsymbol{B}, \boldsymbol{A}]) \\
&(\mathrm{d} g, X, Y) \\
&- f^{2} g(\boldsymbol{I} \otimes[\boldsymbol{B}, \boldsymbol{A}[\boldsymbol{B}, \boldsymbol{A}]]-\boldsymbol{A} \otimes[\boldsymbol{B},[\boldsymbol{B}, \boldsymbol{A}]]-\boldsymbol{B} \otimes \boldsymbol{A}[\boldsymbol{B}, \boldsymbol{A}]+\boldsymbol{A} \boldsymbol{B} \otimes[\boldsymbol{B}, \boldsymbol{A}]) \\
&(\mathrm{d} g, Y, X) .
\end{aligned}
$$

Formula (25) can be derived by a direct (although cumbersome) calculation.

From Definition 9, by means of some algebraic manipulations one can derive another useful result.

Lemma 16. Let $M$ be a differentiable manifold, $f \in C^{\infty}(M)$ and let $\boldsymbol{A}, \boldsymbol{B}: \mathfrak{X}(M) \rightarrow$ $\mathfrak{X}(M)$ be two $(1,1)$-tensors. Then, for all $f \in C^{\infty}(M)$ we have

$$
\begin{aligned}
\mathcal{H}_{f \boldsymbol{I}+\boldsymbol{A}, \boldsymbol{B}}(X, Y)= & \mathcal{H}_{\boldsymbol{A}, \boldsymbol{B}}(X, Y)+(\boldsymbol{I} \otimes \boldsymbol{B}+\boldsymbol{B} \otimes \boldsymbol{I})[\boldsymbol{A}, \boldsymbol{B}](\mathrm{d} f, X, Y) \\
& -(\boldsymbol{I} \otimes \boldsymbol{B}+\boldsymbol{B} \otimes \boldsymbol{I})[\boldsymbol{A}, \boldsymbol{B}](\mathrm{d} f, Y, X) .
\end{aligned}
$$

The following result clarifies the geometric meaning of the Haantjes bracket of level 2.

Lemma 17. Let $M$ be a differentiable manifold and $\boldsymbol{A}, \boldsymbol{B}: \mathfrak{X}(M) \rightarrow \mathfrak{X}(M)$ two commuting $(1,1)$-tensors which can be simultaneously diagonalized in a local chart of $M$. Then, for any $X, Y \in \mathfrak{X}(M)$, the Haantjes bracket $\mathcal{H}_{A, B}(X, Y)$ vanishes.

Proof. We denote by $\boldsymbol{A}_{i}^{i}$ and $\boldsymbol{B}_{j}^{j}$ the non-vanishing components of $\boldsymbol{A}$ and $\boldsymbol{B}$, respectively. Then, in a local chart where the operators diagonalize simultaneously, using Eq. (15), by means of a direct calculation we get

$$
\left(\mathcal{H}_{\boldsymbol{A}, \boldsymbol{B}}\right)_{j k}^{i}=\llbracket \boldsymbol{A}, \boldsymbol{B} \rrbracket_{j k}^{i}\left(2 \boldsymbol{A}_{i}^{i} \boldsymbol{B}_{i}^{i}+\boldsymbol{A}_{j}^{j} \boldsymbol{B}_{k}^{k}+\boldsymbol{A}_{k}^{k} \boldsymbol{B}_{j}^{j}-\boldsymbol{A}_{i}^{i}\left(\boldsymbol{B}_{k}^{k}+\boldsymbol{B}_{j}^{j}\right)-\boldsymbol{B}_{i}^{i}\left(\boldsymbol{A}_{k}^{k}+\boldsymbol{A}_{j}^{j}\right)\right),
$$

where $\llbracket \boldsymbol{A}, \boldsymbol{B} \rrbracket_{j k}^{i}$ is explicitly given in formula (6). If $i, j, k$ are all different, then $\llbracket \boldsymbol{A}, \boldsymbol{B} \rrbracket_{j k}^{i}=0$. Moreover, if $i=k \neq j$ or $i=j \neq k$, the sum in the r.h.s. vanishes as well.

The latter property, which does not hold in the case of the Frölicher-Nijenhuis bracket, is analogous to the one valid for the standard Haantjes torsion of diagonalizable operators. In fact, their Haantjes torsion vanishes, whereas the Nijenhuis one does not necessarily. 
3.3. Haantjes brackets and Haantjes modules. In the following analysis, we shall further illustrate the algebraic relevance of Haantjes brackets of level 2. Indeed they play a crucial role in the study of the $C^{\infty}(M)$-modules of Haantjes operators, that we shall call Haantjes modules.

Definition 18. A Haantjes module is a pair $\left(M, \mathscr{H}_{\mathcal{M}}\right)$ which satisfies the following conditions:

- $M$ is a differentiable manifold of dimension $n$;

- $\mathscr{H}_{\mathcal{M}}$ is a set of Haantjes operators $\boldsymbol{K}: \mathfrak{X}(M) \rightarrow \mathfrak{X}(M)$ such that

$$
\mathcal{H}_{\left(f \boldsymbol{K}_{\mathbf{1}}+g \boldsymbol{K}_{2}\right)}(X, Y)=\mathbf{0}, \quad \forall X, Y \in \mathfrak{X}(M), \quad \forall f, g \in C^{\infty}(M), \quad \forall \boldsymbol{K}_{1}, \boldsymbol{K}_{2} \in \mathscr{H}_{\mathcal{M}} .
$$

Thus, a Haantjes module is a free module of Haantjes operators over the ring of smooth functions on $M$. If property (26) is satisfied only when $f, g$ are real constants, we shall use the denomination of Haantjes vector space.

We determine now the tensorial compatibility conditions ensuring the existence of the Haantjes module generated by two Haantjes operators $\boldsymbol{A}, \boldsymbol{B}: \mathfrak{X}(M) \rightarrow \mathfrak{X}(M)$. First, we construct these conditions in full generality, namely for non-semisimple, non-commuting Haantjes operators. Then, we shall restrict to the important case of semisimple, commuting operators, which arises for instance in Hamiltonian classical mechanics, in the discussion of integrable systems [21,22].

3.3.1. The general case We shall start our analysis with the following identity, valid for all $f, g \in C^{\infty}(M), X, Y \in \mathfrak{X}(M)$ :

$$
\mathcal{H}_{f \boldsymbol{A}+g \boldsymbol{B}}(X, Y)=f^{4} \mathcal{H}_{\boldsymbol{A}}(X, Y)+g^{4} \mathcal{H}_{\boldsymbol{B}}(X, Y)+\sigma_{f \boldsymbol{A}, g \boldsymbol{B}}(X, Y),
$$

where

$$
\sigma_{\boldsymbol{A}, \boldsymbol{B}}(X, Y):=\mathcal{H}_{\boldsymbol{A}, \boldsymbol{B}}(X, Y)+\mathcal{H}_{1}(\boldsymbol{A}, \boldsymbol{B})(X, Y)+\mathcal{H}_{2}(\boldsymbol{A}, \boldsymbol{B})(X, Y)+\mathcal{H}_{2}(\boldsymbol{B}, \boldsymbol{A})(X, Y)
$$

and $\mathcal{H}_{1}(\boldsymbol{A}, \boldsymbol{B}), \mathcal{H}_{2}(\boldsymbol{A}, \boldsymbol{B})$ are the vector valued 2 -forms

$$
\begin{aligned}
& \mathcal{H}_{1}(\boldsymbol{A}, \boldsymbol{B})(X, Y):=\boldsymbol{B}^{2} \tau_{\boldsymbol{A}}(X, Y)+\tau_{\boldsymbol{A}}(\boldsymbol{B} X, \boldsymbol{B} Y)-\boldsymbol{B}\left(\tau_{\boldsymbol{A}}(\boldsymbol{B} X, Y)+\tau_{\boldsymbol{A}}(X, \boldsymbol{B} Y)\right) \\
& \quad+\boldsymbol{A}^{2} \tau_{\boldsymbol{B}}(X, Y)+\tau_{\boldsymbol{B}}(\boldsymbol{A} X, \boldsymbol{A} Y)-\boldsymbol{A}\left(\tau_{\boldsymbol{B}}(\boldsymbol{A} X, Y)+\tau_{\boldsymbol{B}}(X, \boldsymbol{A} Y)\right), \\
& \mathcal{H}_{2}(\boldsymbol{A}, \boldsymbol{B})(X, Y):=(\boldsymbol{A B}+\boldsymbol{B} \boldsymbol{A}) \tau_{\boldsymbol{A}}(X, Y)+\tau_{\boldsymbol{A}}(\boldsymbol{A} X, \boldsymbol{B} \boldsymbol{Y})+\tau_{\boldsymbol{A}}(\boldsymbol{B} X, \boldsymbol{A} Y) \\
& \quad-\boldsymbol{A}\left(\tau_{\boldsymbol{A}}(\boldsymbol{B} X, Y)+\tau_{\boldsymbol{A}}(X, \boldsymbol{B} Y)\right)-\boldsymbol{B}\left(\tau_{\boldsymbol{A}}(\boldsymbol{A} X, Y)+\tau_{\boldsymbol{A}}(X, \boldsymbol{A} Y)\right) \\
& \quad+\boldsymbol{A}^{2} \llbracket \boldsymbol{A}, \boldsymbol{B} \rrbracket(X, Y)+\llbracket \boldsymbol{A}, \boldsymbol{B} \rrbracket(\boldsymbol{A} X, \boldsymbol{A} Y)-\boldsymbol{A}(\llbracket \boldsymbol{A}, \boldsymbol{B} \rrbracket(\boldsymbol{A} X, Y)+\llbracket \boldsymbol{A}, \boldsymbol{B} \rrbracket(X, \boldsymbol{A} Y)) .
\end{aligned}
$$

They represent new, auxiliary brackets which complement the role of the Haantjes bracket $\mathcal{H}_{\boldsymbol{A}, \boldsymbol{B}}$.

Now, some technical results are in order. Let us introduce the three vector-valued 3tensors

$$
\begin{aligned}
& \mathbf{T}_{i}: \mathfrak{X}^{*}(M) \times \mathfrak{X}(M) \times \mathfrak{X}(M) \rightarrow \mathfrak{X}(M),(\alpha, X, Y) \mapsto \mathbf{T}_{i}(\alpha, X, Y), \quad i=1,2,3: \\
& \mathbf{T}_{1}(\boldsymbol{A}, \boldsymbol{B})(\alpha, X, Y):=\left(\left(\mathbf{T}(\boldsymbol{A}, \boldsymbol{B})+\mathbf{T}^{\prime}(\boldsymbol{A}, \boldsymbol{B})\right)[\boldsymbol{A}, \boldsymbol{B}]\right)(\alpha, X, Y),
\end{aligned}
$$




$$
\begin{aligned}
& \mathbf{T}_{2}(\boldsymbol{A}, \boldsymbol{B})(\alpha, X, Y):=\left(\left(\mathbf{T}(\boldsymbol{A}, \boldsymbol{A})+\mathbf{T}^{\prime}(\boldsymbol{A}, \boldsymbol{A})\right)[\boldsymbol{A}, \boldsymbol{B}]\right)(\alpha, X, Y), \\
& \mathbf{T}_{3}(\boldsymbol{A}, \boldsymbol{B})(\alpha, X, Y):=\left(\left(\mathbf{T}(\boldsymbol{B}, \boldsymbol{B})+\mathbf{T}^{\prime}(\boldsymbol{B}, \boldsymbol{B})\right)[\boldsymbol{A}, \boldsymbol{B}]\right)(\alpha, X, Y),
\end{aligned}
$$

where $\mathbf{T}(\boldsymbol{A}, \boldsymbol{B})$ is defined in Eq. (8) and

$$
\mathbf{T}^{\prime}(\boldsymbol{A}, \boldsymbol{B})(\alpha, X, Y):=(\boldsymbol{A B} \otimes \boldsymbol{I}-\boldsymbol{A} \otimes \boldsymbol{B})(\alpha, X, Y) .
$$

These brackets satisfy the relations

$$
\mathbf{T}_{1}(\boldsymbol{A}, \boldsymbol{B})=-\mathbf{T}_{1}(\boldsymbol{B}, \boldsymbol{A}), \quad \mathbf{T}_{3}(\boldsymbol{A}, \boldsymbol{B})=-\mathbf{T}_{2}(\boldsymbol{B}, \boldsymbol{A}) .
$$

Lemma 19. Let $\boldsymbol{A}, \boldsymbol{B}: \mathfrak{X}(M) \rightarrow \mathfrak{X}(M)$ be two operators. For all $f, g \in C^{\infty}(M)$ and $X, Y \in \mathfrak{X}(M)$, the following identities hold:

$$
\begin{aligned}
& \mathcal{H}_{f \boldsymbol{A}, g \boldsymbol{B}}(X, Y)+\mathcal{H}_{1}(f \boldsymbol{A}, g \boldsymbol{B})(X, Y)=f^{2} g^{2}\left(\mathcal{H}_{\boldsymbol{A}, \boldsymbol{B}}(X, Y)+\mathcal{H}_{1}(\boldsymbol{A}, \boldsymbol{B})(X, Y)\right) \\
& \quad+f g^{2}\left(\boldsymbol{T}_{1}(\boldsymbol{A}, \boldsymbol{B})-\boldsymbol{T}_{1}^{T}(\boldsymbol{A}, \boldsymbol{B})\right)(\mathrm{d} f, X, Y)-f^{2} g\left(\boldsymbol{T}_{1}(\boldsymbol{A}, \boldsymbol{B})-\boldsymbol{T}_{1}^{T}(\boldsymbol{A}, \boldsymbol{B})\right)(\mathrm{d} g, X, Y) \\
& \left.\left.\mathcal{H}_{2}(f \boldsymbol{A}, g \boldsymbol{B})(X, Y)=f^{3} g \mathcal{H}_{2}(\boldsymbol{A}, \boldsymbol{B})(X, Y)\right)+f^{2} g\left(\boldsymbol{T}_{2}(\boldsymbol{A}, \boldsymbol{B})-\boldsymbol{T}_{2}^{T}(\boldsymbol{A}, \boldsymbol{B})\right)(\mathrm{d} f, Y, X)\right) \\
& \quad-f^{3}\left(\boldsymbol{T}_{2}(\boldsymbol{A}, \boldsymbol{B})-\boldsymbol{T}_{2}^{T}(\boldsymbol{A}, \boldsymbol{B})\right)(\mathrm{d} g, X, Y) \\
& \left.\left.\mathcal{H}_{2}(g \boldsymbol{B}, f \boldsymbol{A})(X, Y)=f g^{3} \mathcal{H}_{2}(\boldsymbol{B}, \boldsymbol{A})(X, Y)\right)+g^{3}\left(\boldsymbol{T}_{3}(\boldsymbol{A}, \boldsymbol{B})-\boldsymbol{T}_{3}^{T}(\boldsymbol{A}, \boldsymbol{B})\right)(\mathrm{d} f, Y, X)\right) \\
& \quad-f g^{2}\left(\boldsymbol{T}_{3}(\boldsymbol{A}, \boldsymbol{B})-\boldsymbol{T}_{3}^{T}(\boldsymbol{A}, \boldsymbol{B})\right)(\mathrm{d} g, X, Y) .
\end{aligned}
$$

From Eq. (34) we get the identity

$$
\begin{aligned}
\sigma_{f \boldsymbol{A}, g \boldsymbol{B}}(X, Y)= & f^{2} g^{2}\left(\mathcal{H}(\boldsymbol{A}, \boldsymbol{B})(X, Y)+\mathcal{H}_{1}(\boldsymbol{A}, \boldsymbol{B})(X, Y)\right) \\
+ & f^{3} g \mathcal{H}_{2}(\boldsymbol{A}, \boldsymbol{B})(X, Y)+f g^{3} \mathcal{H}_{2}(\boldsymbol{B}, \boldsymbol{A})(X, Y) \\
+ & \left(\left(g^{3}\left(\mathbf{T}_{3}-\mathbf{T}_{3}^{T}\right)+g^{2} f\left(\mathbf{T}_{1}-\mathbf{T}_{1}^{T}\right)+g f^{2}\left(\mathbf{T}_{2}-\mathbf{T}_{2}^{T}\right)\right)(\boldsymbol{A}, \boldsymbol{B})\right) \\
& (\mathrm{d} f, X, Y) \\
+ & \left(\left(f^{3}\left(\mathbf{T}_{3}-\mathbf{T}_{3}^{T}\right)+f^{2} g\left(\mathbf{T}_{1}-\mathbf{T}_{1}^{T}\right)+f g^{2}\left(\mathbf{T}_{2}-\mathbf{T}_{2}^{T}\right)\right)(\boldsymbol{B}, \boldsymbol{A})\right) \\
& (\mathrm{d} g, X, Y) .
\end{aligned}
$$

From Eqs. (27), (35) and (33), taking into account the previous discussion, we obtain our tensorial characterization of Haantjes modules.

Theorem 20. Let $M$ be a differentiable manifold and let $\boldsymbol{A}, \boldsymbol{B}: \mathfrak{X}(M) \rightarrow \mathfrak{X}(M)$ be two Haantjes operators. Then, $\boldsymbol{A}$ and $\boldsymbol{B}$ generate a Haantjes module if and only if the differential conditions

$$
\mathcal{H}_{\boldsymbol{A}, \boldsymbol{B}}+\mathcal{H}_{1}(\boldsymbol{A}, \boldsymbol{B})=\mathbf{0}, \quad \mathcal{H}_{2}(\boldsymbol{A}, \boldsymbol{B})=\mathbf{0}, \quad \mathcal{H}_{2}(\boldsymbol{B}, \boldsymbol{A})=\mathbf{0},
$$

together with the algebraic conditions

$$
\mathbf{T}_{1}(\boldsymbol{A}, \boldsymbol{B})=\mathbf{T}_{1}^{T}(\boldsymbol{A}, \boldsymbol{B}), \quad \mathbf{T}_{2}(\boldsymbol{A}, \boldsymbol{B})=\mathbf{T}_{2}^{T}(\boldsymbol{A}, \boldsymbol{B}), \quad \mathbf{T}_{2}(\boldsymbol{B}, \boldsymbol{A})=\mathbf{T}_{2}^{T}(\boldsymbol{B}, \boldsymbol{A})
$$


are satisfied. In particular, $\boldsymbol{A}$ and $\boldsymbol{B}$ generate a Haantjes vector space if and only if the differential conditions (36) are fulfilled.

Corollary 21. Let $\boldsymbol{A}$ and $\boldsymbol{B}$ be two commuting Haantjes operators. They generate a Haantjes module if and only if conditions (36) are fulfilled.

Some examples of applications are in order.

- Haantjes moduli: In [22], the Haantjes algebra generated by the operators $\left\{\boldsymbol{I}, \boldsymbol{K}_{2}^{(P W)}\right.$, $\boldsymbol{K}_{3}^{(P W)}$ \} for the Post-Winternitz superintegrable system has been constructed. This algebra has rank 4 , with a basis given by $\left\{\boldsymbol{I}, \boldsymbol{K}_{2}^{(P W)}, \boldsymbol{K}_{3}^{(P W)}, \boldsymbol{K}_{2}^{(P W)} \boldsymbol{K}_{3}^{(P W)}\right\}$. It admits a submodule, whose basis is provided by the subset of non-commuting operators $\left\{\boldsymbol{K}_{2}^{(P W)}, \boldsymbol{K}_{3}^{(P W)}\right\}$, which fulfill conditions (36) and (37).

- Haantjes vector spaces: In [8], (2+1)-dimensional hydrodynamic type systems of the form $u_{t}=\boldsymbol{A}(u) u_{x}+\boldsymbol{B}(u) u_{y}$, where $u=u(x, y, t)$ have been considered. In the case of the generalized Benney system and of an isoentropic gas, the two associated operators $\boldsymbol{A}(u)$ and $\boldsymbol{B}(u)$ do not commute. Also, they fulfill Eq. (36) but not Eq. (37). Therefore, these operators generate a Haantjes vector space.

3.3.2. The semisimple, Abelian case In the previous analysis, the Haantjes operators $\boldsymbol{A}$ and $\boldsymbol{B}$ are not supposed to be semisimple. Let us show that if $\boldsymbol{A}$ and $\boldsymbol{B}$ commute and they are semisimple, then the three differential conditions (36) reduce to the vanishing of the Haantjes bracket $\mathcal{H}_{\boldsymbol{A}, \boldsymbol{B}}$. Although this is a special case of the previous construction, it requires an ad hoc analysis.

To this aim, we need to evaluate the Frölicher-Nijenhuis bracket, as well as the brackets $\mathcal{H}_{\boldsymbol{A}, \boldsymbol{B}}, \mathcal{H}_{1}(\boldsymbol{A}, \boldsymbol{B})$ and $\mathcal{H}_{2}(\boldsymbol{A}, \boldsymbol{B})$ over two common eigenvectors $X_{\mu}$ and $Y_{v}$ of two (arbitrary) operators $\boldsymbol{A}$ and $\boldsymbol{B}$ (the details of the calculation are reported in Appendix 6.3).

Proposition 22. Let $\boldsymbol{A}$ and $\boldsymbol{B}$ two Haantjes operators and $X_{\mu}, Y_{v}$ two common eigenvectors. Then

$$
\mathcal{H}_{1}(\boldsymbol{A}, \boldsymbol{B})\left(X_{\mu}, Y_{v}\right)=\mathbf{0} .
$$

In addition, if $\boldsymbol{A}$ and $\boldsymbol{B}$ also commute, then

$$
\mathcal{H}_{2}(\boldsymbol{A}, \boldsymbol{B})\left(X_{\mu}, Y_{v}\right)=\mathbf{0}, \quad \mathcal{H}_{2}(\boldsymbol{B}, \boldsymbol{A})\left(X_{\mu}, Y_{\nu}\right)=\mathbf{0} .
$$

Proof. From Eq. (84) in Appendix 6.3 and the assumption that $\boldsymbol{A}$ and $\boldsymbol{B}$ are Haantjes operators, it follows that

$$
\left[X_{\mu}, Y_{\nu}\right] \in\left(\operatorname{ker}\left(\boldsymbol{A}-\mu_{1} \boldsymbol{I}\right) \oplus \operatorname{ker}\left(\boldsymbol{A}-v_{1} \boldsymbol{I}\right)\right) \cap\left(\operatorname{ker}\left(\boldsymbol{B}-\mu_{2} \boldsymbol{I}\right) \oplus \operatorname{ker}\left(\boldsymbol{B}-v_{2} \boldsymbol{I}\right)\right),
$$

where the subindices 1 and 2 refer to the operators $\boldsymbol{A}$ and $\boldsymbol{B}$, respectively.

Consequently, it is evident from Eq. (87) that Eq. (38) holds for any pair of Haantjes operators $\boldsymbol{A}$ and $\boldsymbol{B}$. In addition, if $\boldsymbol{A}$ and $\boldsymbol{B}$ commute, from Eqs. (87) it follows that Eqs. (39) also hold.

We can now formulate our main result concerning the characterization of Haantjes modules.

Theorem 23. Let $\boldsymbol{A}, \boldsymbol{B}: \mathfrak{X}(M) \rightarrow \mathfrak{X}(M)$ be two commuting semisimple Haantjes operators. They generate a Haantjes module if and only if

$$
\mathcal{H}_{\boldsymbol{A}, \boldsymbol{B}}(X, Y)=\mathbf{0}, \quad \forall X, Y \in \mathfrak{X}(M) .
$$


Proof. As $\boldsymbol{A}$ and $\boldsymbol{B}$ are semisimple commuting operators, they share a local eigenframe. In this eigenframe, the two operators take simultaneously a diagonal form. Since they are also Haantjes operators, all of the brackets $\mathcal{H}_{1}(\boldsymbol{A}, \boldsymbol{B}), \mathcal{H}_{2}(\boldsymbol{A}, \boldsymbol{B})$ and $\mathcal{H}_{2}(\boldsymbol{B}, \boldsymbol{A})$ identically vanish as a consequence of Proposition 22. Thus, from Corollary 21 the result follows.

3.4. Spectral analysis. At this stage, we wish to discuss some of the spectral properties of non-semisimple operators on a manifold from the perspective of the theory of higherlevel Nijenhuis torsions. Let us denote by $\operatorname{Spec}(\boldsymbol{A}):=\left\{\lambda_{1}(\boldsymbol{x}), \lambda_{2}(\boldsymbol{x}), \ldots, \lambda_{s}(\boldsymbol{x})\right\}$ the set of the distinct eigenvalues of an operator $\boldsymbol{A}: \mathfrak{X}(M) \rightarrow \mathfrak{X}(M)$. In the forthcoming considerations, we shall always assume that these eigenvalues are real and pointwise distinct. We denote by

$$
\mathcal{D}_{i}(\boldsymbol{x}):=\operatorname{ker}\left(\boldsymbol{A}(\boldsymbol{x})-\lambda_{i}(\boldsymbol{x}) \boldsymbol{I}\right)^{\rho_{i}}, \quad i=1, \ldots, s
$$

the $i$-th generalized eigen-distribution of index $\rho_{i}$, that is the distribution of all the generalized eigenvectors corresponding to the eigenvalue $\lambda_{i}$. In Eq. (42), $\rho_{i}$ stands for the Riesz index of $\lambda_{i}$, which is the minimum integer such that

$$
\operatorname{ker}\left(\boldsymbol{A}(\boldsymbol{x})-\lambda_{i}(\boldsymbol{x}) \boldsymbol{I}\right)^{\rho_{i}} \equiv \operatorname{ker}\left(\boldsymbol{A}(\boldsymbol{x})-\lambda_{i}(\boldsymbol{x}) \boldsymbol{I}\right)^{\rho_{i}+1}
$$

we also assume that $\rho_{i}$ is (locally) independent of $\boldsymbol{x}$. When $\rho_{i}=1, \mathcal{D}_{i}$ is a proper eigendistribution. Hereafter, unless differently stated, we shall use the adjective "generalized" to include the case of proper eigen-distributions as well.

In several applications, it is also useful to consider the action of our higher-level torsions on the generalized eigenvectors of $\boldsymbol{A}$. Inspired by a formula for the Nijenhuis torsion evaluated on eigenvectors (proved in Appendix 6.2), we construct a generalized expansion, in terms of commutators, for the torsions of any level. It can be proved by induction over the integers $m \geq 2$.

Proposition 24. Let $\boldsymbol{A}$ be a $(1,1)$-tensor and $X_{\alpha}, Y_{\beta}$ be two of its generalized eigenvectors of $\mathcal{D}_{\mu}, \mathcal{D}_{\nu}$, respectively (see formulae (81) and (82)). Then, for any integer $m \geq 2$ the following formula holds:

$$
\tau_{\boldsymbol{A}}^{(m)}\left(X_{\alpha}, Y_{\beta}\right)=\sum_{i, j=0}^{m}(-1)^{i+j}\left(\begin{array}{c}
m \\
i
\end{array}\right)\left(\begin{array}{c}
m \\
j
\end{array}\right)(\boldsymbol{A}-\mu \mathbf{I})^{m-i}(\boldsymbol{A}-\nu \mathbf{I})^{m-j}\left[X_{\alpha-i}, Y_{\beta-j}\right] .
$$

This proposition will be useful in the proof of Lemmas 36 and 38, stated below.

\section{Generalized Nijenhuis Torsions and Haantjes Brackets for Nilcyclic Operators}

In order to clarify the geometric relevance of both the generalized Nijenhuis torsions and the Haantjes brackets of level $m$, we shall focus first on the case of nilcyclic operators (namely operators which are both nilpotent and cyclic). According to the classical Jordan-Chevalley decomposition theorem, given a vector space $V$, any linear endomorphism $\boldsymbol{L}: V \rightarrow V$ with real eigenvalues can be written in a unique way as the sum 
$\boldsymbol{L}=\boldsymbol{D}+\boldsymbol{N}$, where $\boldsymbol{D}$ is a diagonalizable operator and $\boldsymbol{N}$ is a nilpotent one, commuting with $\boldsymbol{D}$. In turn, the nilpotent operator $\boldsymbol{N}$ can be decomposed as the direct sum of nilcyclic operators.

Hereafter, the symbol \langle\rangle will denote a $C^{\infty}(M)$-linear span of vector fields.

Definition 25 (Natural flag). Let $\left(U, x^{1}, \ldots, x^{n}\right)$ be a local coordinate chart and $\left(\frac{\partial}{\partial x^{1}}, \ldots, \frac{\partial}{\partial x^{n}}\right)$ the natural reference frame associated with it. The flag of integrable distributions

$$
\begin{aligned}
\mathcal{C}_{0} & =\{0\} \subset \mathcal{C}_{1}=<e_{1}>\subset \mathcal{C}_{2}=<e_{1}, e_{2}>\subset \cdots \subset \mathcal{C}_{n-1} \\
& =<e_{1}, \ldots, e_{n-1}>\subset \mathcal{C}_{n}=\mathfrak{X}(U),
\end{aligned}
$$

where $e_{i}:=\frac{\partial}{\partial x^{i}}(i=1, \ldots, n-1)$, will be called the natural flag associated with the local chart $\left(x^{1}, \ldots, x^{n}\right)$.

4.1. Triangular form of nilcyclic operators. Let $M$ be an $n$-dimensional differentiable manifold, and $\boldsymbol{A}: \mathfrak{X}(M) \rightarrow \mathfrak{X}(M)$ be a nilcyclic [5] operator, that is a nilpotent $(1,1)$ tensor of maximal index $n$ :

$$
\boldsymbol{A}^{n}=\mathbf{0} \text { and } \boldsymbol{A}^{n-1} \neq \mathbf{0} .
$$

This condition implies that there exist local reference frames, possibly non integrable ones, in which $\boldsymbol{A}$ is represented by a single, upper strictly triangular Jordan block. Under these assumptions, the characteristic null flag of $\boldsymbol{A}$

$$
\{\mathbf{0}\} \subset \operatorname{ker} \boldsymbol{A} \subset \operatorname{ker} \boldsymbol{A}^{2} \subset \cdots \subset \operatorname{ker} \boldsymbol{A}^{n}=\mathfrak{X}(M)
$$

is a complete flag [14], that is, $\operatorname{rank}\left(\operatorname{ker} \boldsymbol{A}^{j}\right)=j, j=1, \ldots, n$. Also, the following inclusions hold:

$$
\boldsymbol{A}^{k}\left(\operatorname{ker} \boldsymbol{A}^{j}\right) \subseteq \operatorname{ker} \boldsymbol{A}^{j-k}, \quad j \geq k=1, \ldots, n .
$$

Let us assume that there exists a local coordinate chart $\left(x^{1}, \ldots, x^{n}\right)$ on $M$ where $\boldsymbol{A}$ takes the upper strictly triangular form

$$
\boldsymbol{A}=\sum_{i, j=1}^{n} a_{j}^{i}(\boldsymbol{x}) \frac{\partial}{\partial x^{i}} \otimes \mathrm{d} x^{j}, \quad a_{j}^{i}=0 \quad \text { if } i \geq j .
$$

Here $a_{j}^{i}(\boldsymbol{x})=a_{j}^{i}\left(x^{1}, \ldots, x^{n}\right)$ are smooth arbitrary functions depending on the local coordinates on $M$. In this case, the integrable distributions of the natural flag coincide with the kernels of the powers of the operator $\boldsymbol{A}$. Precisely,

$$
\mathcal{C}_{j}=\operatorname{ker} \boldsymbol{A}_{\mid U}^{j}, \quad j=1, \ldots, n .
$$

The following result establishes a necessary condition for a nilcyclic operator to be represented in the upper triangular form, in a suitable coordinate chart.

Proposition 26. Let $M$ be an n-dimensional differentiable manifold, and $\boldsymbol{A}: \mathfrak{X}(M) \rightarrow$ $\mathfrak{X}(M)$ be a nilcyclic $(1,1)$-tensor on $M$. If there exists a local chart where the operator A takes the triangular form (46), then the generalized Nijenhuis torsion of level $(k-1)$ vanishes for all $X, Y \in \operatorname{ker} A^{k}$ :

$$
\tau_{\boldsymbol{A}}^{(k-1)}\left(\operatorname{ker} \boldsymbol{A}^{k}, \operatorname{ker} \boldsymbol{A}^{k}\right)=\mathbf{0}, \quad k \in \mathbb{N} \backslash\{0,1\} .
$$


Proof. First, we observe that the (strong) invariance conditions

$$
\boldsymbol{A}^{p}\left(\mathcal{C}_{j}\right) \subseteq \mathcal{C}_{j-p}, \quad p=0, \ldots, n,
$$

hold as a consequence of relations (45) and (47). In the latter conditions, it is understood that $\mathcal{C}_{j-p} \equiv \mathcal{C}_{0}$ for $j \leq p$.

Then, we can proceed by induction over $k=2, \ldots, n-1$. To this aim, notice that for $k=2$, we have

$$
\begin{aligned}
\tau_{\boldsymbol{A}}\left(e_{1}, e_{2}\right) & =\boldsymbol{A}^{2}\left[e_{1}, e_{2}\right]+\left[\boldsymbol{A} e_{1}, \boldsymbol{A} e_{2}\right]-\boldsymbol{A}\left(\left[\boldsymbol{A} e_{1}, e_{2}\right]+\left[e_{1}, \boldsymbol{A} e_{2}\right]\right) \\
& =-\boldsymbol{A}\left[e_{1}, \boldsymbol{A} e_{2}\right]=\mathbf{0} .
\end{aligned}
$$

The first addend vanishes because both $e_{1}, e_{2}$ are constant fields, the second and third one vanish because $e_{1} \in \operatorname{ker} \boldsymbol{A}$; the last term is zero due to both the invariance condition (49) and the involutivity of ker $\boldsymbol{A}$, which is a distribution of rank 1 . Now we assume that

$$
\tau_{\boldsymbol{A}}^{(k-1)}\left(e_{i}, e_{j}\right)=\mathbf{0}, \quad i, j=1, \ldots, k .
$$

This hypothesis, jointly with Definition 11 and the $\boldsymbol{A}$-invariance of ker $\boldsymbol{A}^{k}$ implies

$$
\tau_{\boldsymbol{A}}^{(k)}\left(e_{i}, e_{j}\right)=\mathbf{0}, \quad i, j=1, \ldots, k
$$

We are left with the terms

$$
\tau_{\boldsymbol{A}}^{(k)}\left(e_{i}, e_{k+1}\right), \quad i=1, \ldots, k,
$$

which can be evaluated by means of Eq. (17). We obtain

$$
\tau_{\boldsymbol{A}}^{(k)}\left(e_{i}, e_{k+1}\right)=\sum_{p, q=0}^{k}(-1)^{-(p+q)}\left(\begin{array}{l}
k \\
p
\end{array}\right)\left(\begin{array}{l}
k \\
q
\end{array}\right) \boldsymbol{A}^{p+q}\left[\boldsymbol{A}^{k-p} e_{i}, \boldsymbol{A}^{k-q} e_{k+1}\right] i=1, \ldots k .
$$

As $e_{i} \in \operatorname{ker} \boldsymbol{A}^{k}$, the addends corresponding to $p=0$ vanish. Moreover, for $p>0$, by virtue of Eq. (49), the following inclusions hold:

$$
\begin{aligned}
\boldsymbol{A}^{p+q}\left[\boldsymbol{A}^{k-p} e_{i}, \boldsymbol{A}^{k-q} e_{k+1}\right] & \subseteq \boldsymbol{A}^{p+q}\left[\mathcal{C}_{i-(k-p)}, \mathcal{C}_{k+1-(k-q)}\right] \subseteq \boldsymbol{A}^{p+q}\left(\mathcal{C}_{\max (i-k+p, 1+q)}\right) \\
& \subseteq \mathcal{C}_{-(p+q)+\max (i-k+p, 1+q)}=\mathcal{C}_{0}
\end{aligned}
$$

We can now infer a direct, but important consequence of Proposition (26).

Corollary 27. Let $M$ be an $n$-dimensional differentiable manifold, $n \geq 2$ and $\boldsymbol{A}$ : $\mathfrak{X}(M) \rightarrow \mathfrak{X}(M)$ be a nilcyclic $(1,1)$-tensor on $M$. Then, the condition

$$
\tau_{\boldsymbol{A}}^{(n-1)}(X, Y)=\mathbf{0}, \quad X, Y \in \mathfrak{X}(M),
$$

is necessary for the existence of a local chart where A takes the triangular form (46).

Proof. It is sufficient to apply Proposition (26) to the torsion of level $k=n$ and to observe that ker $\boldsymbol{A}^{n}=\mathfrak{X}(M)$, as $\boldsymbol{A}$ is nilcyclic. 
Consider the slightly more general case of a tensor of the form

$$
\boldsymbol{L}=\lambda \boldsymbol{I}+\boldsymbol{A}, \quad \lambda \in C^{\infty}(M),
$$

where $\boldsymbol{A}$ is a nilcyclic operator. We have the following result.

Corollary 28. Let $M$ be an $n$-dimensional differentiable manifold, $n \geq 3$ and $\boldsymbol{L}$ : $\mathfrak{X}(M) \rightarrow \mathfrak{X}(M)$ be a $(1,1)$-tensor of the form (53). If there exists a local chart where $\boldsymbol{L}$ takes the triangular form

$$
\boldsymbol{L}=\sum_{i, j=1}^{n}\left(\lambda(\boldsymbol{x}) \delta_{j}^{i}+a_{j}^{i}(\boldsymbol{x})\right) \frac{\partial}{\partial x^{i}} \otimes \mathrm{d} x^{j}, \quad a_{j}^{i}=0 \quad \text { if } i \geq j
$$

then

$$
\tau_{\boldsymbol{L}}^{(k-1)}(X, Y)=\mathbf{0}, \quad \forall X, Y \in \operatorname{ker}(\boldsymbol{L}-\lambda \boldsymbol{I})^{k}, \quad 3 \leq k \leq n-1,
$$

and

$$
\tau_{\boldsymbol{L}}^{(n-1)}(X, Y)=\mathbf{0}, \quad \forall \quad X, Y \in \mathfrak{X}(M)
$$

Proof. The previous relations hold as a consequence of Proposition 26, Corollary 27 and Corollary 14.

4.2. An open problem: a Jordan-Chevalley decomposition. The relevance of the higherlevel Nijenhuis torsions in the study of nilcyclic operators suggests, in a natural way, an interesting problem: namely, to ascertain whether there exists a Jordan-Chevalley-type decomposition for generic operators. Precisely, we propose the following, general

Problem. Let $M$ be an $n$-dimensional differentiable manifold. Determine under which conditions there exist coordinate charts on $M$ such that an operator field $\boldsymbol{L}$ : $\mathfrak{X}(M) \rightarrow \mathfrak{X}(M)$ can be decomposed into the sum of two operators $\boldsymbol{L}=\boldsymbol{D}+\boldsymbol{N}$, where $\boldsymbol{D}$ is a diagonal operator and $\boldsymbol{N}$ is an upper strictly triangular operator, commuting with $D$.

4.3. Conjecture for higher Haantjes brackets. Inspired by the previous discussion, we conjecture the following result (which has been tested in many examples).

Conjecture 29. Let $M$ be an $n$-dimensional differentiable manifold, and $\boldsymbol{A}, \boldsymbol{B}: \mathfrak{X}(M) \rightarrow$ $\mathfrak{X}(M)$ be two nilpotent commuting $(1,1)$-tensors on $M$. The vanishing of their generalized Haantjes bracket of level $(n-1)$

$$
\mathcal{H}_{\boldsymbol{A}, \boldsymbol{B}}^{(n-1)}(X, Y)=\mathbf{0}
$$

is a necessary condition for the existence of a local chart where the tensors $\boldsymbol{A}, \boldsymbol{B}$ take simultaneously the triangular form (46). 


\section{Frobenius Integrability and a Generalized Haantjes Theorem}

5.1. Integrability of eigen-distributions: necessary and sufficient conditions. We shall illustrate now the role of the new families of generalized torsions introduced in this paper in the detection of the properties of integrability of the generalized eigen-distributions admitted by a generic $(1,1)$-tensor.

In the first part of this discussion, the eigenvalues and eigenvectors of operators are supposed to be known. However, this hypothesis will be removed in the statement of our main theorem: indeed, no knowledge a priori of the spectrum and the eigen-distributions of the operators involved will be assumed.

Remark 30. All the eigen-distributions considered are supposed to be regular, that is they have constant rank on $M$. For involutive distributions, this condition is equivalent to their Frobenius integrability.

Definition 31. Let us consider a set of distributions $\left\{\mathcal{D}_{i}, \mathcal{D}_{j}, \ldots, \mathcal{D}_{k}\right\}$. We shall say that such distributions are mutually integrable if

(i) each of them is integrable;

(ii) any sum $\mathcal{D}_{i}+\mathcal{D}_{j}+\cdots+\mathcal{D}_{k}$ is also integrable.

First we state the following

Lemma 32. Let $\boldsymbol{A}: \mathfrak{X}(M) \rightarrow \mathfrak{X}(M)$ be a non-invertible operator. For any $X, Y \in$ ker $\boldsymbol{A}$, we have

$$
\tau_{\boldsymbol{A}}^{(m)}(X, Y)=\boldsymbol{A}^{2 m}[X, Y], \quad m \in \mathbb{N} \backslash\{0\} .
$$

Proof. Equation (58) comes from Eq. (17) taking into account that the terms with $p<m$ and $q<m$ vanish.

Let us recall that the Riesz index of a non-invertible operator $\boldsymbol{A}$ is the Riesz index $\rho$ of its zero eigenvalue (supposed to be constant in an open dense subset of $M$ ), namely the minimum integer $\rho$ that makes stationary the sequence

$$
\{0\} \subset \operatorname{ker} \boldsymbol{A} \subset \operatorname{ker} \boldsymbol{A}^{2} \subset \cdots \subset \operatorname{ker} \boldsymbol{A}^{\rho}=\operatorname{ker} \boldsymbol{A}^{\rho+j} \subseteq \mathfrak{X}(M), \quad j \in \mathbb{N} .
$$

Proposition 33. Let $\boldsymbol{A}: \mathfrak{X}(M) \rightarrow \mathfrak{X}(M)$ be an operator and $\rho$ its Riesz index. The following conditions are equivalent:

(1) the distribution $\operatorname{ker} \boldsymbol{A}^{\rho}$ is involutive;

$$
\exists m \in \mathbb{N} \backslash\{0\} \text { such that } \tau_{\boldsymbol{A}^{\rho}}^{(m)}\left(\operatorname{ker} \boldsymbol{A}^{\rho}, \operatorname{ker} \boldsymbol{A}^{\rho}\right)=\mathbf{0} ;
$$

$$
\forall m \in \mathbb{N} \backslash\{0\}, \quad \tau_{\boldsymbol{A}^{\rho}}^{(m)}\left(\operatorname{ker} \boldsymbol{A}^{\rho}, \operatorname{ker} \boldsymbol{A}^{\rho}\right)=\mathbf{0} .
$$

Proof. 1) $\Longleftrightarrow 2$ ). From Eq. (58) applied to $\boldsymbol{A}^{\rho}$, we get

$$
\tau_{\boldsymbol{A}^{\rho}}^{(m)}\left(\operatorname{ker} \boldsymbol{A}^{\rho}, \operatorname{ker} \boldsymbol{A}^{\rho}\right)=\boldsymbol{A}^{2 \rho m}\left[\operatorname{ker} \boldsymbol{A}^{\rho}, \operatorname{ker} \boldsymbol{A}^{\rho}\right] .
$$

Consequently,

$$
\left[\operatorname{ker} \boldsymbol{A}^{\rho}, \operatorname{ker} \boldsymbol{A}^{\rho}\right] \subseteq \operatorname{ker} \boldsymbol{A}^{2 \rho m} \stackrel{(59)}{=} \operatorname{ker} \boldsymbol{A}^{\rho}
$$

if and only if the 1.h.s. of Eq. (62) vanishes for some $m \in \mathbb{N} \backslash\{0\}$.

$1) \Longrightarrow 3$ ) It is a direct consequence of Eq. (62). The converse statement can be proved by following the same reasoning used in the proof of the first equivalence. 
The equivalence of the conditions (2) and (3) can be geometrically interpreted by observing that, if the distribution $\mathcal{D}=\operatorname{ker} \boldsymbol{A}^{\rho}$ is integrable, then the operator $\boldsymbol{A}^{\rho}$ can be restricted to each integral leaf of $\mathcal{D}$; besides, each of these restricted operators vanishes.

Thus, applying Proposition 33 to each operator $\boldsymbol{B}_{i}:=\boldsymbol{A}-\lambda_{i} \boldsymbol{I}$, we obtain a novel necessary and sufficient condition for the integrability of the generalized eigen-distributions of an operator with real eigenvalues.

Corollary 34. Let $\boldsymbol{A}: \mathfrak{X}(M) \rightarrow \mathfrak{X}(M)$ be an operator and $\mathcal{D}_{i}=\operatorname{ker}\left(\boldsymbol{A}-\lambda_{i} I\right)^{\rho_{i}}$, where $\lambda_{i} \in \operatorname{Spec}(\boldsymbol{A})$. Then, the distribution $\mathcal{D}_{i}$ is involutive if and only if there exists $m \in \mathbb{N} \backslash\{0\}$, such that

$$
\tau_{\left(\boldsymbol{A}-\lambda_{i} \boldsymbol{I}\right)^{\rho_{i}}}^{(m)}\left(\mathcal{D}_{i}, \mathcal{D}_{i}\right)=\mathbf{0} .
$$

Remark 35. The original Nijenhuis theorem [19] was not stated in the general case of non-semisimple operators. However, the previous analysis allows us to conclude that both the Nijenhuis torsion and the higher-level ones are equally valid, from a theoretical point of view, to detect the integrability properties of the generalized eigen-distributions of a non-semisimple operator.

5.2. Main theorem. The results stated above provide new necessary and sufficient conditions for the integrability of eigen-distributions of generalized eigenvectors. However, as we have remarked, they require the knowledge a priori of the eigenvalues and eigenvectors of the considered operator. Instead, in the spirit of the seminal theorems by Nijenhuis and Haantjes, it is desirable to have integrability conditions which do not require to solve explicitly eigenvalue problems, since this task becomes computationally intractable for large values of $n$. To this aim, we shall propose a novel strategy, based on the notion of higher-level Nijenhuis torsions.

Formally, the problem we shall address is the following: to establish the conditions ensuring a priori the integrability of the generalized eigen-distributions of an operator $\boldsymbol{A}$ whose Haantjes torsion does not vanish, without recurring to the explicit determination of its eigen-distributions. To the best of our knowledge, no result is known regarding this problem. In the main theorem stated below, we will offer a solution to this problem by introducing a family of sufficient conditions for integrability.

First, let us prove some preliminary results.

Lemma 36. Let $\boldsymbol{A}: \mathfrak{X}(M) \rightarrow \mathfrak{X}(M)$ be an operator, $\mu \in \operatorname{Spec}(\boldsymbol{A})$ and $X_{\alpha}, Y_{\beta} \in \mathcal{D}_{\mu}$ two of its generalized eigenvectors, of index $\alpha, \beta$ respectively, belonging to (possibly different) Jordan chains (see formulae (81) and (82)). If there exists an integer $m \geq 1$ such that

then we have:

$$
\tau_{\boldsymbol{A}}^{(m)}\left(\mathcal{D}_{\mu}, \mathcal{D}_{\mu}\right)=\mathbf{0}
$$

$$
\left[X_{\alpha}, Y_{\beta}\right] \in \operatorname{ker}(\boldsymbol{A}-\mu \mathbf{I})^{\alpha+\beta+m}=\operatorname{ker}(\boldsymbol{A}-\mu \mathbf{I})^{\min \left(\alpha+\beta+m, \rho_{\mu}\right)} \subseteq \operatorname{ker}(\boldsymbol{A}-\mu \mathbf{I})^{\rho_{\mu}},
$$

where $\min (\cdot, \cdot)$ stands for the minimum of its arguments.

Proof. First, we prove the case $m \geq 2$. If $\alpha=\beta=1$ and $\mu=v$, Eq. (44) implies that $\left[X_{1}, Y_{1}\right] \in \operatorname{ker}(\boldsymbol{A}-\mu \mathbf{I})^{2 m}$. By induction over $(\alpha+\beta)$ and applying the operator $(\boldsymbol{A}-\mu \mathbf{I})^{\alpha+\beta-m}$ to both members of Eq. (44), it follows that

$$
\left[X_{\alpha}, Y_{\beta}\right] \in \operatorname{ker}(\boldsymbol{A}-\mu \mathbf{I})^{\alpha+\beta+m} \text {. }
$$


In order to prove the case $m=1$, we observe that if the Nijenhuis torsion $\tau_{\boldsymbol{A}}^{(1)}$ vanishes over the vector fields of $\mathcal{D}_{\mu}$, then $\tau_{\boldsymbol{A}}^{(m)}$ vanishes as well, for $m \geq 1$.

Proposition 37. Let $\boldsymbol{A}: \mathfrak{X}(M) \rightarrow \mathfrak{X}(M)$ be an operator. Each of its generalized eigendistributions $\mathcal{D}_{\mu}$ with Riesz index $\rho_{\mu} \geq 1$ is involutive if

$$
\tau_{\boldsymbol{A}}^{(m)}\left(\mathcal{D}_{\mu}, \mathcal{D}_{\mu}\right)=\mathbf{0}
$$

for some integer $m \geq 1$. In addition, in the semisimple case $\left(\rho_{\mu}=1\right)$, if $\mathcal{D}_{\mu}$ is involutive, then condition (66) is fulfilled for each integer $m \geq 2$.

Proof. Assuming condition (66), Lemma 36 immediately implies that $\mathcal{D}_{\mu}$ is an involutive distribution, since

$$
\left[\mathcal{D}_{\mu}, \mathcal{D}_{\mu}\right] \subseteq \mathcal{D}_{\mu}
$$

In the specific case $\rho_{\mu}=1$, every $\mu$-eigenvector of $\boldsymbol{A}$ is a proper eigenvector, and from Eq. (44) for $m \geq 2$ one infers that

$$
\tau_{\boldsymbol{A}}^{(m)}\left(\mathcal{D}_{\mu}, \mathcal{D}_{\mu}\right)=0 \Longleftrightarrow\left[X_{1}, Y_{1}\right] \in \operatorname{ker}(\boldsymbol{A}-\mu \mathbf{I})^{2 m}=\operatorname{ker}(\boldsymbol{A}-\mu \mathbf{I})=\mathcal{D}_{\mu} .
$$

We deduce that for $\rho_{\mu}=1$, condition (66) is also necessary for the involutivity of $\mathcal{D}_{\mu}$, for $m \geq 2$.

Lemma 38. Let $\boldsymbol{A}: \mathfrak{X}(M) \rightarrow \mathfrak{X}(M)$ be an operator and $\mathcal{D}_{\mu}, \mathcal{D}_{\nu}$ two eigen-distributions satisfying, for some integer $m \geq 1$, the condition

$$
\tau_{\boldsymbol{A}}^{(m)}\left(\mathcal{D}_{\mu}, \mathcal{D}_{\nu}\right)=\mathbf{0}
$$

Then, the commutator of two generalized eigenvectors of $\boldsymbol{A}$ with respect to two different eigenvalues $\mu$, $v$, satisfies the property

$$
\begin{aligned}
{\left[X_{\alpha}, Y_{\beta}\right] } & \in \operatorname{ker}(\boldsymbol{A}-\mu \mathbf{I})^{\alpha+m-1} \oplus \operatorname{ker}(\boldsymbol{A}-\nu \mathbf{I})^{\beta+m-1} \\
& =\operatorname{ker}(\boldsymbol{A}-\mu \mathbf{I})^{\min \left(\alpha+m-1, \rho_{\mu}\right)} \oplus \operatorname{ker}(\boldsymbol{A}-\nu \mathbf{I})^{\min \left(\beta+m-1, \rho_{\nu}\right)} \\
& \subseteq \operatorname{ker}(\boldsymbol{A}-\mu \mathbf{I})^{\rho_{\mu}} \oplus \operatorname{ker}(\boldsymbol{A}-\nu \mathbf{I})^{\rho_{\nu}}
\end{aligned}
$$

with $1 \leq \alpha \leq \rho_{\mu}, 1 \leq \beta \leq \rho_{\nu}$.

Proof. If $\alpha=\beta=1$ and $\mu \neq v$, Eq. (44) for $m \geq 2$ implies that

$$
\left[X_{1}, Y_{1}\right] \in \operatorname{ker}(\boldsymbol{A}-\mu \mathbf{I})^{m} \oplus \operatorname{ker}(\boldsymbol{A}-\nu \mathbf{I})^{m} \text {. }
$$

By induction over $(\alpha+\beta)$, the result follows for $m \geq 2$ applying the operator $(\boldsymbol{A}-$ $\mu \mathbf{I})^{\alpha-1}(\boldsymbol{A}-\nu \mathbf{I})^{\beta-1}$ to both members of Eq. (44). If $\tau_{\boldsymbol{A}}^{(1)}\left(\mathcal{D}_{\mu}, \mathcal{D}_{\nu}\right)=0$, we also have $\tau_{\boldsymbol{A}}^{(m)}\left(\mathcal{D}_{\mu}, \mathcal{D}_{\nu}\right)=0$ for $m \geq 1$. This completes the proof.

The latter Lemma also implies $\left[\mathcal{D}_{\mu}, \mathcal{D}_{\nu}\right] \subset \mathcal{D}_{\mu} \oplus \mathcal{D}_{\nu}$. This observation ensures the validity of the next result. 
Proposition 39. Let $\boldsymbol{A}: \mathfrak{X}(M) \rightarrow \mathfrak{X}(M)$ be an operator and $\mathcal{D}_{\mu}, \mathcal{D}_{\nu}$ two eigendistributions with Riesz indices $\rho_{\mu}, \rho_{v}$ respectively. Assume that for some $m \geq 1$,

$$
\tau_{\boldsymbol{A}}^{(m)}\left(\mathcal{D}_{\mu}, \mathcal{D}_{\nu}\right)=\mathbf{0}
$$

Then the distribution

$$
\mathcal{D}_{\mu} \oplus \mathcal{D}_{\nu} \equiv \operatorname{ker}(\boldsymbol{A}-\mu \mathbf{I})^{\rho_{\mu}} \oplus \operatorname{ker}(\boldsymbol{A}-v \mathbf{I})^{\rho_{\nu}}, \quad \mu \neq v
$$

is involutive.

Now, we can prove our main result concerning the mutual integrability of the eigendistributions of operators.

Theorem 40. Let $\boldsymbol{A}: \mathfrak{X}(M) \rightarrow \mathfrak{X}(M)$ be an operator. Assume that

$$
\tau_{\boldsymbol{A}}^{(m)}(X, Y)=\mathbf{0}, \quad X, Y \in \mathfrak{X}(M)
$$

for some $m \geq 1$. Then, each generalized eigen-distribution of $\boldsymbol{A}$ as well as each direct sum of its eigen-distributions is integrable.

Proof. This result is a direct consequence of Propositions 37 and 39 whose hypotheses are indeed fulfilled once we assume the validity of condition (71).

5.3. Block-diagonalization. As a nontrivial application of Theorem (40), we shall prove that given an operator $\boldsymbol{A}$, condition (71) is also sufficient to ensure the existence of a local chart where the operator $\boldsymbol{A}$ can be block-diagonalized. Potentially relevant applications can be found, for instance, in the theory of hydrodynamic-type systems [3], in the study of partial separability of Hamiltonian systems [6] and, more generally, in the context of Courant's problems for first-order hyperbolic systems of partial differential equations [7].

Let $\boldsymbol{A}$ be an operator satisfying condition (71); we denote by $r_{i}$ the rank of the distribution $\mathcal{D}_{i}$ of $\boldsymbol{A}$. We also introduce the distribution (of corank $r_{i}$ )

$$
\mathcal{E}_{i}:=\operatorname{Im}\left(\boldsymbol{A}-\lambda_{i} \mathbf{I}\right)^{\rho_{i}}=\bigoplus_{j=1, j \neq i}^{s} \mathcal{D}_{j}, \quad i=1, \ldots, s
$$

which is spanned by all the generalized eigenvectors of $\boldsymbol{A}$, except those associated with the eigenvalue $\lambda_{i}$ (we remind that $\boldsymbol{A}$ by hypothesis has real eigenvalues). We shall say that $\mathcal{E}_{i}$ is a characteristic distribution of $\boldsymbol{A}$. Let $\mathcal{E}_{i}^{\circ}$ denote the annihilator of the distribution $\mathcal{E}_{i}$. The cotangent spaces of $M$ can be decomposed as

$$
T_{x}^{*} M=\bigoplus_{i=1}^{s} \mathcal{E}_{i}^{\circ}(\boldsymbol{x}) .
$$

As a consequence of Theorem 40, each characteristic distribution $\mathcal{E}_{i}$ is integrable. We shall denote by $\mathrm{E}_{i}$ the foliation associated with $\mathcal{E}_{i}$ and by $E_{i}(\boldsymbol{x})$ the connected leave through $\boldsymbol{x}$, belonging to $\mathrm{E}_{i}$. Thus, given the set of distributions $\left\{\mathcal{E}_{1}, \mathcal{E}_{2}, \ldots, \mathcal{E}_{s}\right\}$, we have associated an equal number of foliations $\left\{\mathrm{E}_{1}, \mathrm{E}_{2}, \ldots, \mathrm{E}_{s}\right\}$. This set of foliations is referred to as the characteristic web of $\boldsymbol{A}$ and the leaves $E_{i}(\boldsymbol{x})$ of each foliation $\mathrm{E}_{i}$ as the characteristic fibers of the web. 
Definition 41. Let $\boldsymbol{A}: \mathfrak{X}(M) \rightarrow \mathfrak{X}(M)$ be an operator satisfying Eq. (71). A collection of $r_{i}$ smooth functions will be said to be adapted to the foliation $\mathrm{E}_{i}$ of the characteristic web of $\boldsymbol{A}$ if the level sets of such functions coincide with the characteristic fibers of $\mathrm{E}_{i}$.

Definition 42. Let $\boldsymbol{A}: \mathfrak{X}(M) \rightarrow \mathfrak{X}(M)$ be an operator satisfying Eq. (71). A parametrization of the characteristic web of $\boldsymbol{A}$ is an ordered set of $n$ independent smooth functions listed as $\left(f^{1}, \ldots, f^{i}, \ldots, f^{s}\right)$, such that for any $i=1, \ldots, s$, the ordered subset $f^{i}=\left(f^{i, 1}, \ldots, f^{i, r_{i}}\right)$ is adapted to the $i$-th characteristic foliation of the web:

$$
f_{\mid E_{i}(\mathbf{x})}^{i, k}=c^{i, k} \quad \forall E_{i}(\mathbf{x}) \in \mathrm{E}_{i}, \quad k=1, \ldots, r_{i} .
$$

Here $c^{i, k}$ are real constants depending on the indices $i$ and $k$ only. In this case, we shall say that the collection of these functions is adapted to the web and that each of them is a characteristic function.

Proposition 43. Let $\boldsymbol{A}: \mathfrak{X}(M) \rightarrow \mathfrak{X}(M)$ be an operator. If

$$
\tau_{\boldsymbol{A}}^{(m)}(X, Y)=\mathbf{0}, \quad X, Y \in \mathfrak{X}(M)
$$

for some $m \geq 1$, then $\boldsymbol{A}$ admits local charts where it takes a block-diagonal form.

Proof. Theorem 40 ensures that each characteristic distribution $\mathcal{E}_{i}$ is integrable. Thus, we can also deduce the existence of $r_{i}$ exact one-forms $\left(\mathrm{d} x^{i, 1}, \ldots, \mathrm{d} x^{i, r_{i}}\right)$ in the corresponding annihilator $\mathcal{E}_{i}^{\circ}$; consequently, there exist functions $\boldsymbol{x}^{i}=\left(x^{i, 1}, \ldots, x^{i, r_{i}}\right)$ adapted to the characteristic foliation $\mathrm{E}_{i}$. Collecting together all these functions, we get a local chart $\left\{U,\left(\boldsymbol{x}^{1}, \ldots, \boldsymbol{x}^{i}, \ldots, \boldsymbol{x}^{s}\right)\right\}$, adapted to the characteristic web. The natural frame associated $\left\{\frac{\partial}{\partial \boldsymbol{x}^{1}}, \ldots, \frac{\partial}{\partial \boldsymbol{x}^{i}}, \ldots, \frac{\partial}{\partial \boldsymbol{x}^{s}}\right\}$ is a generalized eigen-frame. To prove this, it is sufficient to observe that the following decomposition holds:

$$
\mathcal{D}_{i}^{\circ}=\bigoplus_{j=1, j \neq i}^{s} \mathcal{E}_{j}^{\circ}
$$

Thus, any generalized eigenvector $W \in \mathcal{D}_{i}$ leaves invariant all the coordinate functions except at most the characteristic functions $\boldsymbol{x}^{i}=\left(x^{i, 1}, \ldots, x^{i, r_{i}}\right)$ of $\mathrm{E}_{i}$. Thus, we deduce that

$$
W=W\left(x^{i}\right) \frac{\partial}{\partial \boldsymbol{x}^{i}}=\sum_{k=1}^{r_{i}} W\left(x^{i, k}\right) \frac{\partial}{\partial x^{i, k}} .
$$

Therefore

$$
\mathcal{D}_{i_{\mid U}}=\left\langle\frac{\partial}{\partial x^{i, 1}}, \ldots, \frac{\partial}{\partial x^{i, r_{i}}}\right\rangle \text {. }
$$

This means that each frame equivalent to $\left\{\frac{\partial}{\partial \boldsymbol{x}^{1}}, \ldots, \frac{\partial}{\partial \boldsymbol{x}^{i}}, \ldots, \frac{\partial}{\partial \boldsymbol{x}^{s}}\right\}$ is an integrable eigenframe of generalized eigenvectors. Consequently, there exists an equivalence class of integrable frames, with their local charts associated. In these charts, the operator $\boldsymbol{A}$, due to the invariance of its eigen-distributions, takes a block-diagonal form. 
5.4. A comparison with Haantjes's classical theorem. Let $\boldsymbol{A}: \mathfrak{X}(M) \rightarrow \mathfrak{X}(M)$ be an operator with real eigenvalues. In his seminal paper [11], Haantjes proved the following, fundamental theorem:

(i) If $\boldsymbol{A}$ is a semisimple operator, the vanishing of its Haantjes torsion

$$
\mathcal{H}_{A}(X, Y)=\mathbf{0}, \quad \forall X, Y \in \mathfrak{X}(M)
$$

is a necessary and sufficient condition for the mutual integrability of all of its eigen-distributions.

(ii) If $\boldsymbol{A}$ is non-semisimple, then condition (78) is sufficient to guarantee the mutual integrability of its generalized eigen-distributions, but it is not necessary.

Our improvement of the Haantjes theorem consists in the family of conditions (71), which are more general than the standard vanishing condition of the Haantjes torsion. In fact, given a non-semisimple operator $\boldsymbol{A}$, no conclusion about integrability of its eigendistributions can be deduced from the Haantjes theorem, if $\mathcal{H}_{A}(X, Y) \neq \mathbf{0}$. However, if there exists $m>2$ such that $\tau_{\boldsymbol{A}}^{(m)}(X, Y)=\mathbf{0}$, this weaker condition is sufficient to ensure integrability.

In the semisimple case $\left(\rho_{i}=1 \forall i=1, \ldots, s\right)$, we recover Haantjes's result on integrability directly from Proposition 37 . Instead, in the most general, non-semisimple case $\left(\rho_{i}>1\right)$, Theorem 40 provides an infinite family of new sufficient conditions.

The following, simple example can illustrate the potential relevance of Theorem 40 in applicative contexts. Indeed, already in the case $n=3$ a generic non-semisimple operator is not necessarily a Haantjes one. Therefore, the Haantjes theorem does not apply. However, in our example, the associated generalized torsion of level three vanishes; this guarantees mutual integrability.

Example 44. Let $M$ be a 3-dimensional manifold and $\left(x^{1}, x^{2}, x^{3}\right)$ a local chart in $M$. Consider the operator

$$
\begin{aligned}
\boldsymbol{L}(\boldsymbol{x})= & \lambda_{1}(\boldsymbol{x})\left(\frac{\partial}{\partial x^{1}} \otimes \mathrm{d} x^{1}+\frac{\partial}{\partial x^{2}} \otimes \mathrm{d} x^{2}\right)+\lambda_{2}(\boldsymbol{x}) \frac{\partial}{\partial x^{3}} \otimes \mathrm{d} x^{3} \\
& +f(\boldsymbol{x}) \frac{\partial}{\partial x^{1}} \otimes \mathrm{d} x^{2}+g(\boldsymbol{x}) \frac{\partial}{\partial x^{2}} \otimes \mathrm{d} x^{3},
\end{aligned}
$$

with $\lambda_{1}, \lambda_{2}, f, g \in C^{\infty}(M), \lambda_{1} \neq \lambda_{2}$. A direct calculation shows that, for generic choices of these functions, the Nijenhuis and Haantjes torsions do not vanish identically; however, $\tau_{\boldsymbol{L}}^{(3)}(X, Y)=\mathbf{0}$. Therefore, according to Theorem 40, the generalized eigendistributions of $\boldsymbol{L}$ are mutually integrable. To construct them explicitly, observe that the minimal polynomial of $\boldsymbol{L}$ is $m(\lambda):=\left(\lambda-\lambda_{1}\right)^{2}\left(\lambda-\lambda_{2}\right)$, so that the Riesz indices of $\lambda_{1}$ and $\lambda_{2}$ are $\rho_{1}=2, \rho_{2}=1$, respectively. We obtain the generalized eigen-distribution $\mathcal{D}_{1}=\operatorname{ker}\left(\boldsymbol{L}-\lambda_{i} \boldsymbol{I}\right)^{2}=\left\langle\frac{\partial}{\partial x^{1}}, \frac{\partial}{\partial x^{2}}\right\rangle$, which is trivially integrable, as well as the proper eigen-distribution $\mathcal{D}_{2}=\operatorname{ker}\left(\boldsymbol{L}-\lambda_{2} \boldsymbol{I}\right)=\left\langle X_{\lambda_{2}}\right\rangle$, with

$$
X_{\lambda_{2}}=f g \frac{\partial}{\partial x^{1}}+\left(\lambda_{2}-\lambda_{1}\right) g \frac{\partial}{\partial x^{2}}+\left(\lambda_{1}-\lambda_{2}\right)^{2} \frac{\partial}{\partial x^{3}} .
$$

The latter eigen-distribution is of rank 1 and obviously integrable. Thus, as $\mathcal{D}_{1}=\mathcal{E}_{2}$ and $\mathcal{D}_{2}=\mathcal{E}_{1}$, we get the spectral decompositions of the tangent spaces $T_{\boldsymbol{x}} M=\mathcal{D}_{1} \oplus \mathcal{E}_{1}=$ 
$\mathcal{D}_{2} \oplus \mathcal{E}_{2}$. Correspondingly, for the cotangent spaces, we obtain $T_{x}^{*} M=\mathcal{E}_{1}^{\circ} \oplus \mathcal{E}_{2}^{\circ}$, where the annihilators of the characteristic distributions of $\boldsymbol{L}$ are

$$
\mathcal{E}_{1}^{\circ}=\left\langle\left(\lambda_{1}-\lambda_{2}\right)^{2} \mathrm{~d} x^{1}-f g \mathrm{~d} x^{3},\left(\lambda_{1}-\lambda_{2}\right) \mathrm{d} x^{1}+f \mathrm{~d} x^{2}\right\rangle, \quad \mathcal{E}_{2}^{\circ}=\left\langle\mathrm{d} x^{3}\right\rangle .
$$

In order to construct explicitly a local chart where $\boldsymbol{L}$ takes a block-diagonal form (as ensured by Proposition 43), let us consider the space $\mathbb{R}^{3}$ endowed with Cartesian coordinates $\left(x^{1}, x^{2}, x^{3}\right)$. We make the simple choice $\lambda_{1}=x^{1}+x^{2}+x^{3}, \lambda_{2}=x^{1}+x^{2}, f=x^{3}$, $g=x^{1}$ in $M=R^{3} \backslash\left\{x^{3}=0\right\}$ (to guarantee $\lambda_{1} \neq \lambda_{2}$ ). By integrating the annihilators of the characteristic distributions (as explained in the proof of Proposition 43), we find the local coordinate chart

$$
y^{1}=x^{1}+x^{2}, \quad y^{2}=\frac{x^{1}}{x^{3}}, \quad y^{3}=x^{3} .
$$

On this chart, the operator $\boldsymbol{L}$ takes the block-diagonal form

$$
\boldsymbol{L}=\left[\begin{array}{cc|c}
y^{1}+2 y^{3} & -\left(y^{3}\right)^{2} & 0 \\
1 & y^{1} & 0 \\
\hline 0 & 0 & y^{1}
\end{array}\right]
$$

As we have shown, in the case of non-semisimple operators, the criterion of the vanishing of the Haantjes torsion, being only sufficient, may fail to detect the mutual integrability of the eigen-distributions even for very basic examples. Nevertheless, Theorem 40 provides us with a more general tensorial test, guaranteeing integrability without the need for an explicit analysis of the eigen-distributions involved. Once integrability is ascertained, one can enter this kind of analysis in order to block-diagonalize the considered operator.

Acknowledgements. The authors gratefully thank Prof. Y. Kosmann-Schwarzbach for useful discussions. Also, P. T. wishes to thank heartily Prof. N. Kamran for a careful reading of the manuscript, discussions and encouragement. We also wish to thank the Referees for many helpful suggestions. The research of P. T. has been supported by the research project PGC2018-094898-B-I00, Ministerio de Ciencia, Innovación y Universidades and Agencia Estatal de Investigación, Spain, and by the Severo Ochoa Programme for Centres of Excellence in R\&D (CEX2019-000904-S), Ministerio de Ciencia, Innovación y Universidades y Agencia Estatal de Investigación, Spain. The research of G. T. has been supported by the research project FRA2020-2021, Universitá degli Studi di Trieste, Italy. P. T. is member of the Gruppo Nazionale di Fisica Matematica (GNFM).

Funding Open Access funding provided thanks to the CRUE-CSIC agreement with Springer Nature.

Open Access This article is licensed under a Creative Commons Attribution 4.0 International License, which permits use, sharing, adaptation, distribution and reproduction in any medium or format, as long as you give appropriate credit to the original author(s) and the source, provide a link to the Creative Commons licence, and indicate if changes were made. The images or other third party material in this article are included in the article's Creative Commons licence, unless indicated otherwise in a credit line to the material. If material is not included in the article's Creative Commons licence and your intended use is not permitted by statutory regulation or exceeds the permitted use, you will need to obtain permission directly from the copyright holder. To view a copy of this licence, visit http://creativecommons.org/licenses/by/4.0/.

Publisher's Note Springer Nature remains neutral with regard to jurisdictional claims in published maps and institutional affiliations. 


\section{Appendix}

6.1. On the Haantjes bracket. We propose an explicit formula for the Haantjes bracket of level 2 in terms of commutators of vector fields:

$$
\begin{aligned}
\mathcal{H}_{\boldsymbol{A}, \boldsymbol{B}}(X, Y):=\frac{1}{2}(\boldsymbol{A} \boldsymbol{B}+\boldsymbol{B} \boldsymbol{A})^{2}[X, Y]+\boldsymbol{A} \boldsymbol{B}(2[\boldsymbol{A} X, \boldsymbol{B} Y]+2[\boldsymbol{B} X, \boldsymbol{A} Y]+[\boldsymbol{A} \boldsymbol{B} X, Y] \\
+[X, \boldsymbol{A} \boldsymbol{B} Y])-2 \boldsymbol{A}(\boldsymbol{A} \boldsymbol{B}+\boldsymbol{B} \boldsymbol{A})([\boldsymbol{B} X, Y]+[X, \boldsymbol{B} Y])+\left[\boldsymbol{A}^{2} X, \boldsymbol{B}^{2} Y\right]+[\boldsymbol{A} \boldsymbol{B} X, \boldsymbol{B} \boldsymbol{A} Y] \\
-2 \boldsymbol{A}\left([\boldsymbol{B} \boldsymbol{A} X, \boldsymbol{B} Y]+\left[\boldsymbol{A} X, \boldsymbol{B}^{2} Y\right]+[\boldsymbol{A} \boldsymbol{B} X, \boldsymbol{B} Y]+\left[\boldsymbol{B}^{2} X, \boldsymbol{A} Y\right]+[\boldsymbol{B} X, \boldsymbol{A} \boldsymbol{B} Y]\right. \\
\quad+[\boldsymbol{B} \boldsymbol{X}, \boldsymbol{B} \boldsymbol{A} Y])+\boldsymbol{A}^{2}\left(\left[X, \boldsymbol{B}^{2} Y\right]+2[\boldsymbol{B} X, \boldsymbol{B} Y]+\left[\boldsymbol{B}^{2} X, Y\right]\right) \\
\quad+\text { symmetric terms in } \boldsymbol{A}, \boldsymbol{B} .
\end{aligned}
$$

6.2. The Nijenhuis torsion evaluated over generalized eigenvectors. Let $\boldsymbol{A}: \mathfrak{X}(M) \rightarrow$ $\mathfrak{X}(M)$ be an operator. Without loss of generality, we shall focus only on two eigenvalues of $\boldsymbol{A}, \mu=\mu(\boldsymbol{x})$ and $v=v(\boldsymbol{x}) \in \operatorname{Spec}(\boldsymbol{A})$, possibly coincident. Let us denote by $X_{\alpha}$, $Y_{\beta}$ two generalized eigenvectors, with indices $\alpha$ and $\beta$, associated with $\mu$ and $\nu$ :

$$
X_{\alpha} \in \operatorname{ker}(\boldsymbol{A}-\mu \mathbf{I})^{\alpha} \backslash \operatorname{ker}(\boldsymbol{A}-\mu \mathbf{I})^{(\alpha-1)}, \quad Y_{\beta} \in \operatorname{ker}(\boldsymbol{A}-\nu \mathbf{I})^{\beta} \backslash \operatorname{ker}(\boldsymbol{A}-\nu \mathbf{I})^{(\beta-1)} .
$$

They belong to Jordan chains defined in $\mathcal{D}_{\mu}, \mathcal{D}_{\nu}$, respectively:

$$
\boldsymbol{A} X_{\alpha}=\mu X_{\alpha}+X_{\alpha-1}, \quad \boldsymbol{A} Y_{\beta}=v Y_{\beta}+Y_{\beta-1}, \quad 1 \leq \alpha \leq \rho_{\mu}, \quad 1 \leq \beta \leq \rho_{\nu},
$$

where $X_{0}$ and $Y_{0}$ are, by definition, null vector fields. Evaluating the Nijenhuis torsion on such eigenvectors, we obtain

$$
\begin{aligned}
\tau_{\boldsymbol{A}}\left(X_{\alpha}, Y_{\beta}\right)= & (\boldsymbol{A}-\mu \mathbf{I})(\boldsymbol{A}-v \mathbf{I})\left[X_{\alpha}, Y_{\beta}\right]+(\mu-v)\left(X_{\alpha}(v) Y_{\beta}+Y_{\beta}(\mu) X_{\alpha}\right) \\
& -(\boldsymbol{A}-\mu \mathbf{I})\left[X_{\alpha}, Y_{\beta-1}\right]-(\boldsymbol{A}-v \mathbf{I})\left[X_{\alpha-1}, Y_{\beta}\right]+\left[X_{\alpha-1}, Y_{\beta-1}\right] \\
& -\left(X_{\alpha}(v) Y_{\beta-1}+Y_{\beta-1}(\mu) X_{\alpha}\right)+\left(X_{\alpha-1}(\nu) Y_{\beta}+Y_{\beta}(\mu) X_{\alpha-1}\right) .
\end{aligned}
$$

6.3. Haantjes brackets evaluated over common eigenvectors. Let $\boldsymbol{A}$ and $\boldsymbol{B}$ be two arbitrary (not necessarily Haantjes) operators, and let $X_{\mu}$ and $Y_{v}$ be two common eigenvectors. Precisely, let us consider

$$
X_{\mu} \in \operatorname{ker}\left(\boldsymbol{A}-\mu_{1} \boldsymbol{I}\right) \cap \operatorname{ker}\left(\boldsymbol{B}-\mu_{2} \boldsymbol{I}\right), \quad Y_{v} \in \operatorname{ker}\left(\boldsymbol{A}-v_{1} \boldsymbol{I}\right) \cap \operatorname{ker}\left(\boldsymbol{B}-v_{2} \boldsymbol{I}\right) .
$$

The Frölicher-Nijenhuis bracket satisfies the identity

$$
\begin{aligned}
\llbracket \boldsymbol{A}, \boldsymbol{B} \rrbracket\left(X_{\mu}, Y_{v}\right)= & \left(\left(\boldsymbol{A}-\mu_{1} \boldsymbol{I}\right)\left(\boldsymbol{B}-v_{2} \boldsymbol{I}\right)+\left(\boldsymbol{B}-\mu_{2} \boldsymbol{I}\right)\left(\boldsymbol{A}-v_{1} \boldsymbol{I}\right)\right)\left[X_{\mu}, Y_{\nu}\right] \\
& +\left(\left(\mu_{1}-v_{1}\right) Y\left(\mu_{2}\right)+\left(\mu_{2}-v_{2}\right) Y\left(\mu_{1}\right)\right) X_{\mu} \\
& +\left(\left(\mu_{1}-v_{1}\right) X_{\mu}\left(v_{2}\right)+\left(\mu_{2}-v_{2}\right) X_{v}\left(\mu_{1}\right)\right) Y_{v} .
\end{aligned}
$$


Thus, we get

$$
\begin{aligned}
& \mathcal{H}_{\boldsymbol{A}, \boldsymbol{B}}\left(X_{\mu}, Y\right)=\left(\left(\boldsymbol{A}-\mu_{1} \boldsymbol{I}\right)\left(\boldsymbol{B}-v_{2} \boldsymbol{I}\right)+\left(\boldsymbol{B}-\mu_{2} \boldsymbol{I}\right)\left(\boldsymbol{A}-v_{1} \boldsymbol{I}\right)\right)^{2}\left[X_{\mu}, Y_{v}\right], \\
& \mathcal{H}_{1}(\boldsymbol{A}, \boldsymbol{B})\left(X_{\mu}, Y_{v}\right)=\left(\boldsymbol{B}-\mu_{2} \boldsymbol{I}\right)\left(\boldsymbol{B}-v_{2} \boldsymbol{I}\right)\left(\boldsymbol{A}-\mu_{1} \boldsymbol{I}\right)\left(\boldsymbol{A}-v_{1} \boldsymbol{I}\right)\left[X_{\mu}, Y_{\nu}\right] \\
& \quad+\left(\boldsymbol{A}-\mu_{1} \boldsymbol{I}\right)\left(\boldsymbol{A}-v_{1} \boldsymbol{I}\right)\left(\boldsymbol{B}-\mu_{2} \boldsymbol{I}\right)\left(\boldsymbol{B}-v_{2} \boldsymbol{I}\right)\left[X_{\mu}, Y_{v}\right], \\
& \mathcal{H}_{2}(\boldsymbol{A}, \boldsymbol{B})\left(X_{\mu}, Y_{v}\right) \\
& \quad=\left(\left(\boldsymbol{A}-\mu_{1} \boldsymbol{I}\right)\left(\boldsymbol{B}-v_{2} \boldsymbol{I}\right)+\left(\boldsymbol{B}-\mu_{2} \boldsymbol{I}\right)\left(\boldsymbol{A}-v_{1} \boldsymbol{I}\right)\right)\left(\boldsymbol{A}-\mu_{1} \boldsymbol{I}\right)\left(\boldsymbol{A}-v_{1} \boldsymbol{I}\right)\left[X_{\mu}, Y_{\nu}\right] \\
& \quad+\left(\boldsymbol{A}-\mu_{1} \boldsymbol{I}\right)\left(\boldsymbol{A}-v_{1} \boldsymbol{I}\right)\left(\left(\boldsymbol{A}-\mu_{1} \boldsymbol{I}\right)\left(\boldsymbol{B}-v_{2} \boldsymbol{I}\right)+\left(\boldsymbol{B}-\mu_{2} \boldsymbol{I}\right)\left(\boldsymbol{A}-v_{1} \boldsymbol{I}\right)\right)\left[X_{\mu}, Y_{v}\right] .
\end{aligned}
$$

\section{References}

1. Benenti, S., Chanu, C., Rastelli, G.: Remarks on the connection between the additive separation of the Hamilton-Jacobi equation and the multiplicative separation of the Schröedinger equation. I. The completeness and Robertson conditions. J. Math. Phys. 43, 5183-5222 (2002)

2. Bogoyavlenskij, O.I.: Necessary conditions for existence of non-degenerate Hamiltonian structures. Commun. Math. Phys. 182, 253-290 (1996)

3. Bogoyavlenskij, O.I.: Block-diagonalizability problem for hydrodynamic type systems. J. Math. Phys. 47, 063502 (2006)

4. Bogoyavlenskij, O.I., Reynolds, A.P.: Criterion for existence of a Hamiltonian structure. Regul. Chaotic Dyn. 15, 431-439 (2010)

5. Bowen, R.M., Wang, C.C.: Introduction to Vector and Tensors. Dover, New York (2009)

6. Chanu, C.M., Rastelli, G.: Block-separation of variables: a form of partial separation for natural Hamiltonians. SIGMA 15, 013 (2019)

7. Courant, R., Hilbert, D.: Methods of Mathematical Physics II. Interscience Publishers, New York (1962)

8. Ferapontov, E., Khusnutdinova, K.: The Haantjes tensor and double waves for multi-dimensional systems of hydrodynamic type: a necessary condition for integrability. Proc. R. Soc. A 462, 1197-1219 (2006)

9. Ferapontov, E.V., Marshall, D.G.: Differential-geometric approach to the integrability of hydrodynamics chains: the Haantjes tensor. Math. Ann. 339, 61-99 (2007)

10. Frölicher, A., Nijenhuis, A.: Theory of vector valued differential forms. Part I. Indag. Math. 18, 338-359 (1956)

11. Haantjes, J.: On $X_{n-1}$-forming sets of eigenvectors. Indag. Math. 17, 158-162 (1955)

12. Koláŕ, I., Michor, P.W., Slovák, J.: Natural Operations in Differential Geometry. Springer, Berlin (1993)

13. Kosmann-Schwarzbach, Y.: Beyond recursion operators. In: Kielonowski, P., Odzijewicz, A., Previato, E. (eds) Proceedings of the XXXVI Workshop on Geometric Methods in Physics, Białowieża, Poland, July 2017. Birkhauser (2019). arXiv:1712.08908 (2017)

14. Kreuzer, M., Robbiano, L.: Computational Linear and Commutative Algebra. Springer, Berlin (1993)

15. Magri, F.: Haantjes manifolds. J. Phys. Conf. Ser. 482, 012028 (2014)

16. Magri, F.: Haantjes manifolds and Veselov systems. Theor. Math. Phys. 189, 1486-1499 (2016)

17. Magri, F.: Haantjes manifolds with symmetries. Theor. Math. Phys. 196, 1217-1229 (2018)

18. Newlander, A., Nirenberg, L.: Complex analytic coordinates in almost complex manifolds. Ann. Math. 65, 391-404 (1957)

19. Nijenhuis, A.: $X_{n-1}$-forming sets of eigenvectors. Indag. Math. 54, 200-212 (1951)

20. Nijenhuis, A.: Jacobi-type identities for bilinear differential concomitants of certain tensor fields. I. Indag. Math. 17, 390-397 (1955)

21. Reyes Nozaleda, D., Tempesta, P., Tondo, G.: Classical multiseparable Hamiltonian systems, superintegrability and Haantjes geometry. Commun. Nonlinear Sci. Num. Simul. arXiv:2012.09819 (2021)

22. Tempesta, P., Tondo, G.: Haantjes manifolds and classical integrable systems. Ann. Mat. Pura Appl. arXiv:1405.5118v2, https://doi.org/10.1007/s10231-021-01107-4 (2021)

23. Tempesta, P., Tondo, G.: Haantjes algebras and diagonalization. J. Geom. Phys. 160, 103968 (2021) arXiv: 1710.04522

24. Tondo, G., Tempesta, P.: Haantjes structures for the Jacobi-Calogero model and the Benenti systems. SIGMA 12, 023 (2016)

25. Tondo, G.: Haantjes algebras of the Lagrange top. Theor. Math. Phys. 196, 1366-1379 (2018) 